\title{
A Very Reliable Method for Determination of Absolute Configuration of Chiral Secondary Alcohols by ${ }^{1} \mathrm{H}$ NMR Spectroscopy
}

\author{
Yoshio Takeuchi, * Hidehito Fujisawa, and Ryoji Noyori \\ Faculty of Pharmaceutical Sciences, Toyama Medical and \\ Pharmaceutical University, Sugitani Toyama, 930-0194 Japan and \\ Department of Chemistry and Research Center for Materials Science, \\ Nagoya University, Chikusa Nagoya, 464-8602 Japan
}

\begin{abstract}
Experimental
General: Melting points were measured with a Yanaco micro melting point apparatus and uncorrected. Microanalyses were performed by the Microanalysis Center of Toyama Medical and Pharmaceutical University. Spectroscopic measurements were carried out with the following instruments: optical rotations, JASCO DIP-1000 digital polarimeter; IR spectra, Perkin-Elmer 1600 Series FTIR; mass spectra (MS), JEOL JMS-GCmate; high resolution mass spectra (HRMS), JEOL JMS-AX 505 HAD; ${ }^{1} \mathrm{H}$ NMR spectra, JEOL JNM-GX 270 (270 MHz), Varian Gemini 300 (300 MHz), and Varian Unity $500(500 \mathrm{MHz})$, in $\mathrm{CDCl}_{3}$ with TMS $(=0.00 \mathrm{ppm})$ as an internal standard; ${ }^{2} \mathrm{D}$ NMR spectra, JEOL GSX-400 (61 MHz), in $\mathrm{CHCl}_{3}$ with $\mathrm{CDCl}_{3}(=7.26 \mathrm{ppm})$ as an internal standard. ${ }^{19} \mathrm{~F}$ NMR spectra, JEOL JNM-GX 270 $(254 \mathrm{MHz})$, in $\mathrm{CDCl}_{3}$ with $\mathrm{CFCl}_{3}(=0.00 \mathrm{ppm})$ as an internal standard. Open column chromatography, flash column chromatography, and thin layer chromatography were performed on silica gel [Fuji Silysia BW-200, Kanto chemical silica gel 60N $(0.040-0.050 \mathrm{~mm})$ and Merck 5715, respectively].
\end{abstract}

\section{Preparation of (S)-CFTA-Cl and (R)-CFTA-Cl.}

Preparation of ethyl $\alpha$-cyano-p-tolylacetate

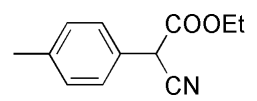

To a solution of $p$-xylyl cyanide $(4.8 \mathrm{~g}, 7 \mathrm{mmol})$ in dry THF $(79 \mathrm{~mL})$ were added $\mathrm{NaH}(60 \%$ dispersion in mineral oil, $2.5 \mathrm{~g}, 60 \mathrm{mmol})$ and diethyl carbonate $(7.3 \mathrm{~mL}, 60 \mathrm{mmol})$. The whole mixture was heated at reflux for $2 \mathrm{~h}$. Water (39 $\mathrm{mL}$ ) was added to the mixture and THF was evaporated. The resulting solution was acidified to $\mathrm{pH} 1$ with conc. $\mathrm{HCl}$ and extracted with AcOEt $(40 \mathrm{~mL} \times 3)$. The combined organic layer was washed with brine, dried over $\mathrm{MgSO}_{4}$, and concentrated in vacuo. The residual oil was purified by column chromatography on silica gel (eluent: hexane/AcOEt $=$ $10 / 1)$ to give ethyl $\alpha$-cyano-p-tolylacetate $(7.4 \mathrm{~g}, 99 \%)$ as a colorless oil. IR (neat): $v=1746(\mathrm{C}=\mathrm{O}), 2252(\mathrm{CN}) \mathrm{cm}^{-1}$; ${ }^{1} \mathrm{H}$ NMR $\left(300 \mathrm{MHz}, \mathrm{CDCl}_{3}\right): \delta=1.28\left(\mathrm{t}, J=7.1 \mathrm{~Hz}, 3 \mathrm{H} ; \mathrm{CH}_{2} \mathrm{CH}_{3}\right.$ ), 2.37 (s, 3H; $\left.\mathrm{Ar}_{-} \mathrm{CH}_{3}\right), 4.24(\mathrm{qd}, J=1.1,7.1 \mathrm{~Hz}, 2 \mathrm{H}$; $\left.\mathrm{CH}_{2}\right), 4.68(\mathrm{~s}, 1 \mathrm{H} ; \mathrm{CH}), 7.22(\mathrm{~d}, J=8.2 \mathrm{~Hz}, 2 \mathrm{H} ; \mathrm{Ar}), 7.35(\mathrm{~d}, J=8.2 \mathrm{~Hz}, 2 \mathrm{H} ; \mathrm{Ar}) ; \mathrm{MS}: m / z: 203\left(\mathrm{M}^{+}\right), 130\left(\mathrm{M}^{+}-\mathrm{CO}_{2} \mathrm{Et}\right)$.

\section{Preparation of ethyl $\alpha$-cyano- $\alpha$-fluoro-p-tolylacetate (( \pm$)$-CFTA ethyl ester)}

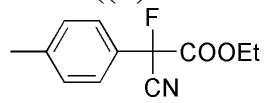

To a solution of ethyl $\alpha$-cyano- $p$-tolylacetate $(3.9 \mathrm{~g}, 19 \mathrm{mmol})$ in dry THF $(100 \mathrm{~mL})$ was added portionwise $\mathrm{NaH}$ (60\% dispersion in mineral oil, $932 \mathrm{mg}, 23 \mathrm{mmol}$ ) at room temperature under nitrogen and the mixture was stirred for 1 $\mathrm{h}$. Into the mixture was introduced diluted $\mathrm{FClO}_{3}$ gas at room temperature for $2 \mathrm{~h}$. Water was added to the reaction mixture and THF was evaporated in vacuo. The resulting solution was acidified to $\mathrm{pH} 1$ with conc. $\mathrm{HCl}$ and extracted with [AcOEt $\left(40 \mathrm{~mL}\right.$ x 3). The combined organic layer was washed with brine, dried over $\mathrm{MgSO}_{4}$, and condensed in vacuo. The residual oil was purified by column chromatography on silica gel (eluent: hexane/AcOEt $=9 / 1)$ to give ethyl $\alpha$-cyano- $\alpha$-fluoro- $p$-tolylacetate $(4.1 \mathrm{~g}, 95 \%)$ as a colorless oil. IR (neat): $v=1774(\mathrm{C}=\mathrm{O}), 2253(\mathrm{CN}) \mathrm{cm}^{-1} ;{ }^{1} \mathrm{H}$ $\operatorname{NMR}\left(300 \mathrm{MHz}, \mathrm{CDCl}_{3}\right): \delta=1.30\left(\mathrm{t}, J=7.3 \mathrm{~Hz}, 3 \mathrm{H} ; \mathrm{CH}_{2} \mathrm{C}_{3}\right), 2.40\left(\mathrm{~s}, 3 \mathrm{H} ; \mathrm{Ar}-\mathrm{CH}_{3}\right) 4.25-4.43\left(\mathrm{~m}, 2 \mathrm{H} ; \mathrm{CH}_{2}\right), 7.29(\mathrm{~d}$, $J=8.2 \mathrm{~Hz}, 2 \mathrm{H} ; \mathrm{Ar}), 7.52(\mathrm{~d}, J=7.8 \mathrm{~Hz}, 2 \mathrm{H} ; \mathrm{Ar}) ;{ }^{19} \mathrm{~F} \mathrm{NMR}\left(254 \mathrm{MHz}, \mathrm{CDCl}_{3}\right): \delta=-144.6(\mathrm{~s}, 1 \mathrm{~F}) ; \mathrm{MS}: m / z: 221\left(\mathrm{M}^{+}\right)$, $148\left(\mathrm{M}^{+}-\mathrm{CO}_{2} \mathrm{Et}\right)$. 


\section{Preparation of ( \pm )- $\alpha$-cyano- $\alpha$-fluoro- $p$-tolylacetyl chloride (( \pm )-CFTA-Cl, 6$)$}

To a solution of $( \pm)$-CFTA ethyl ester $(835 \mathrm{mg}, 3.8 \mathrm{mmol})$ in $\mathrm{THF} / \mathrm{H}_{2} \mathrm{O}(=1 / 1,12 \mathrm{~mL})$ was added $1 \mathrm{M} \mathrm{LiOH}(3.8$ $\mathrm{mL}, 3.8 \mathrm{mmol}$ ) and the mixture was stirred at room temperature for $20 \mathrm{~min}$. After evaporation of THF, the aqueous layer was acidified to $\mathrm{pH} 1$ with conc. $\mathrm{HCl}$ and extracted with $\mathrm{AcOEt}(20 \mathrm{~mL}$ x 3). The combined organic layer was washed with brine and dried over $\mathrm{MgSO}_{4}$. Evaporation of the solvent gave ( \pm )-CFTA (731 mg) as a yellow oil.

To a solution of $( \pm)$-CFTA and DMF $(38 \mathrm{mg}, 0.5 \mathrm{mmol})$ in dry $\mathrm{CH}_{2} \mathrm{Cl}_{2}(30 \mathrm{~mL})$ was added dropwise $(\mathrm{COCl})_{2}(0.66$ $\mathrm{mL}, 7.6 \mathrm{mmol}$ ) and the mixture was stirred at room temperature for $3 \mathrm{~h}$. After evaporation of the excess reagents and solvent, the residual oil was distilled under reduced pressure to give ( \pm )-CFTA-Cl (6) $(519 \mathrm{mg}, 65 \%)$ as a colorless oil. B.p. $56-58{ }^{\circ} \mathrm{C}(0.2 \mathrm{mmHg})$; IR (neat): $v=1794(\mathrm{C}=\mathrm{O}) \mathrm{cm}^{-1} ;{ }^{1} \mathrm{H} \mathrm{NMR}\left(270 \mathrm{MHz}, \mathrm{CDCl}_{3}\right): \delta=2.43\left(\mathrm{~s}, 3 \mathrm{H} ; \mathrm{Ar}-\mathrm{CH}_{3}\right)$, 7.35 (d, $J=8.3 \mathrm{~Hz}, 2 \mathrm{H} ; \mathrm{Ar}), 7.54(\mathrm{~d}, J=8.1 \mathrm{~Hz}, 2 \mathrm{H} ; \mathrm{Ar}) ;{ }^{19} \mathrm{~F} \mathrm{NMR}\left(254 \mathrm{MHz}, \mathrm{CDCl}_{3}\right): \delta=-135.0(\mathrm{~s}, 1 \mathrm{~F})$.

\section{Preparation of $(1 S, 3 R, 4 R, 6 R)-3,7,7-t$ trimethylbicyclo[4.1.0]heptane-3,4-diol (18)}

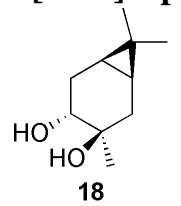

To a solution of MCPBA (>70\%, $25.0 \mathrm{~g}, 101 \mathrm{mmol})$ in $\mathrm{CH}_{2} \mathrm{Cl}_{2}(300 \mathrm{~mL})$ was added (+)-3-carene $(12.4 \mathrm{~g}, 87 \mathrm{mmol})$ and the mixture was stirred at room temperature for $1.5 \mathrm{~h} . \mathrm{Ca}(\mathrm{OH})_{2}(32.0 \mathrm{~g}, 410 \mathrm{mmol})$ was added to the reaction mixture and whole was stirred for $1 \mathrm{~h}$. The white precipitates were removed by filtration through Celite bed and the filtrate was concentrated in vacuo to give a colorless oil. To a stirred mixture of the oil in water $(50 \mathrm{~mL})$ and acetone $(50 \mathrm{~mL})$ was carefully added conc. $\mathrm{H}_{2} \mathrm{SO}_{4}(4$ drops $)$ at $0{ }^{\circ} \mathrm{C}$ and stirring was continued at room temperature for $2 \mathrm{~h}$. After cautious addition of sat. $\mathrm{NaHCO}_{3}(50 \mathrm{~mL})$, most of the organic solvent was evaporated. The residual layer was extracted with AcOEt $\left(70 \mathrm{~mL}\right.$ x 3). The combined organic layer was washed with brine, dried over $\mathrm{Na}_{2} \mathrm{SO}_{4}$, and concentrated. The residual oil was dried well under vacuum to give a brown solid, which was recrystallized from hexane to give the alcohol $(\mathbf{1 8})(11.7 \mathrm{~g}, 79 \%)$ as colorless needles. M.p. $71-76^{\circ} \mathrm{C}$ (from hexane); $[\alpha]_{\mathrm{D}}{ }^{26}-0.4(c=5.0$ in $\left.\mathrm{CHCl}_{3}\right)$; IR (KBr): $v=3376(\mathrm{OH}) \mathrm{cm}^{-1} ;{ }^{1} \mathrm{H}$ NMR $\left(300 \mathrm{MHz}, \mathrm{CDCl}_{3}\right): \delta=0.67-0.75(\mathrm{~m}, 2 \mathrm{H} ;>\mathrm{CH}-), 0.97(\mathrm{~s}, 3 \mathrm{H}$; $\left.\mathrm{CH}_{3}\left(\mathrm{CH}_{3}\right) \mathrm{C}<\right), 1.03\left(\mathrm{~s}, 3 \mathrm{H} ; \mathrm{CH}_{3}\left(\mathrm{CH}_{3}\right) \mathrm{C}<\right), 1.17-1.28\left(\mathrm{~m}, 1 \mathrm{H} ; \mathrm{CH}_{3}(\mathrm{OH}) \mathrm{CC} \underline{\mathrm{H} H C H}<\right), 1.21\left(\mathrm{~s}, 3 \mathrm{H} ; \mathrm{CH}_{3}(\mathrm{OH}) \mathrm{C}<\right), 1.65$ $(\mathrm{ddd}, J=7.7,10.2,14.3 \mathrm{~Hz}, 1 \mathrm{H} ; \mathrm{HOCHC} \underline{\mathrm{HCH}}<), 1.98\left(\mathrm{dd}, J=9.6,14.6 \mathrm{~Hz}, 1 \mathrm{H} ; \mathrm{CH}_{3}(\mathrm{OH}) \mathrm{CCH} \underline{\mathrm{HCH}}<\right), 2.11(\mathrm{dd}, J=$ 7.4, $14.6 \mathrm{~Hz}, 1 \mathrm{H} ; \mathrm{HOCHCH} \underline{\mathrm{HCH}}<), 3.36\left(\mathrm{dd}, J=7.4,10.2 \mathrm{~Hz}, 1 \mathrm{H} ; \mathrm{HOCHCH}_{2}\right)$. MS: $m / z: 170\left(\mathrm{M}^{+}\right)$. Anal. Calcd. for $\mathrm{C}_{12} \mathrm{H}_{18} \mathrm{O}_{2}: \mathrm{C}, 70.55 ; \mathrm{H}, 10.66$. Found: $\mathrm{C}, 70.35 ; \mathrm{H}, 10.39$.

Preparation of $\alpha$-cyano- $\alpha$-fluoro- $p$-tolylacetic acid $(1 R, 2 R, 3 R, 6 S)$-4-hydroxy-4,7,7-trimethyl-bicyclo[4.1.0]hept-3yl esters

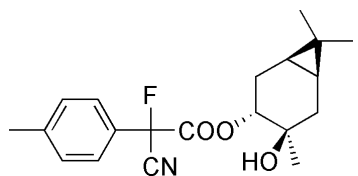

To a mixture of the alcohol (18) $(2.9 \mathrm{~g}, 17 \mathrm{mmol})$ in dry pyridine $(2.5 \mathrm{~mL}, 31 \mathrm{mmol})$ and dry $\mathrm{CH}_{2} \mathrm{Cl}_{2}(20 \mathrm{~mL})$ was added dropwise ( \pm )-CFTA-Cl $(3.3 \mathrm{~g}, 16 \mathrm{mmol})$ at $0{ }^{\circ} \mathrm{C}$ under $\mathrm{N}_{2}$, and the mixture was stirred at room temperature for 5 h. After evaporation of the solvents, excess pyridine was azeotropically removed together with dry toluene $(20 \mathrm{~mL} \times 2)$ to give crude product $(8.6 \mathrm{~g})$. Two diastereomeric esters were separated by column chromatography on silica gel $\left(\mathrm{CH}_{2} \mathrm{Cl}_{2}\right)$ to give $(S)$-CFTA ester of $\mathbf{1 8}(1.7 \mathrm{~g}, 44 \%)$ and $(R)$-CFTA ester of $\mathbf{1 8}(2.0 \mathrm{~g}, 53 \%)$.

Less polar isomer $\left[(S)\right.$-CFTA ester]; yellow needles. M.p. $39-42{ }^{\circ} \mathrm{C} .[\alpha]_{\mathrm{D}}{ }^{28}-17.2(c=2.7$ in $\mathrm{MeOH}) ; \mathrm{IR}(\mathrm{KBr}): v=$ $1760(\mathrm{C}=\mathrm{O}), 2254(\mathrm{CN}), 3341(\mathrm{OH}) \mathrm{cm}^{-1} ;{ }^{1} \mathrm{H}$ NMR $\left(300 \mathrm{MHz}, \mathrm{CDCl}_{3}\right): \delta=0.73-0.79(\mathrm{~m}, 2 \mathrm{H} ;>\mathrm{CH}-), 0.99(\mathrm{~s}, 3 \mathrm{H}$; $\left.\mathrm{C}_{3}\left(\mathrm{CH}_{3}\right) \mathrm{C}<\right), 1.00\left(\mathrm{~s}, 3 \mathrm{H} ; \mathrm{CH}_{3}\left(\mathrm{C}_{3}\right) \mathrm{C}<\right), 1.14$ (s, 3H; $\left.\underline{\mathrm{C}}_{3}(\mathrm{HO}) \mathrm{C}<\right), 1.24-1.32\left(\mathrm{~m}, 1 \mathrm{H} ; \mathrm{CH}_{3}(\mathrm{HO}) \mathrm{CC} \underline{H} H C H<\right), 1.29$ (dd, $\left.J=3.6,14.3 \mathrm{~Hz}, 1 \mathrm{H} ; \mathrm{CH}_{3}(\mathrm{HO}) \mathrm{CCHHCH}<\right), 1.79-1.89$ (m, $\left.1 \mathrm{H} ;-\mathrm{OCHCHHCH}<\right), 2.23$ (dd, $J=7.6,14.2 \mathrm{~Hz}, 1 \mathrm{H}$; $-\mathrm{OCHCH} \underline{\mathrm{HCH}}<$ ), 2.39 (s, 3H; $\left.\mathrm{Ar}_{-} \mathrm{CH}_{3}\right), 4.66(\mathrm{dd}, J=7.8,9.8 \mathrm{~Hz}, 1 \mathrm{H} ; \mathrm{COOCH}), 7.28(\mathrm{~d}, J=8.2 \mathrm{~Hz}, 2 \mathrm{H} ; \mathrm{Ar}), 7.53(\mathrm{~d}$, $J=8.0 \mathrm{~Hz}, 2 \mathrm{H} ; \mathrm{Ar}) ;{ }^{19} \mathrm{~F} \mathrm{NMR}\left(254 \mathrm{MHz}, \mathrm{CDCl}_{3}\right): \delta=-143.48(\mathrm{~s}, 1 \mathrm{~F}) ; \mathrm{MS}: m / z: 345\left(\mathrm{M}^{+}\right)$; Anal. Calcd. for $\mathrm{C}_{20} \mathrm{H}_{24} \mathrm{FNO}_{3}$ : C, 69.55; H, 7.00; N, 4.06. Found: C, 69.59; H, 7.12; N, 4.18.

More polar isomer $\left[(R)\right.$-CFTA ester]; brown oil; $[\alpha]_{\mathrm{D}}{ }^{28}+6.5(c=1.4$ in $\mathrm{MeOH})$; IR (neat): $v=1771(\mathrm{C}=\mathrm{O}), 2254(\mathrm{CN})$, $3431(\mathrm{OH}) \mathrm{cm}^{-1} ;{ }^{1} \mathrm{H}$ NMR $\left(300 \mathrm{MHz}, \mathrm{CDCl}_{3}\right): \delta=0.64-0.79(\mathrm{~m}, 2 \mathrm{H} ;>\mathrm{CH}-), 0.97\left(\mathrm{~s}, 3 \mathrm{H} ; \mathrm{CH}_{3}\left(\mathrm{CH}_{3}\right) \mathrm{C}<\right), 0.99(\mathrm{~s}, 3 \mathrm{H}$; $\left.\mathrm{CH}_{3}\left(\underline{\mathrm{C}}_{3}\right) \mathrm{C}<\right), 1.30\left(\mathrm{~s}, 3 \mathrm{H} ; \underline{\mathrm{C}}_{3}(\mathrm{HO}) \mathrm{C}<\right), 1.35$ (dd, $\left.J=4.5,5.7 \mathrm{~Hz}, 1 \mathrm{H} ; \mathrm{CH}_{3}(\mathrm{HO}) \mathrm{CC} \underline{\mathrm{H} H C H}<\right), 1.55-1.67$ (m, $1 \mathrm{H}$; $-\mathrm{OCHC} \underline{H} H C H<), 1.99-2.10\left(\mathrm{~m}, 1 \mathrm{H} ; \mathrm{CH}_{3}(\mathrm{HO}) \mathrm{CCH} \underline{H C H}<\right), 2.23(\mathrm{dd}, J=7.6,14.2 \mathrm{~Hz}, 1 \mathrm{H} ;-\mathrm{OCHCH} \underline{H C H}<), 2.40(\mathrm{~s}$, 
$\left.3 \mathrm{H} ; \mathrm{Ar}-\mathrm{CH}_{3}\right), 4.71(\mathrm{dd}, J=7.7,9.6 \mathrm{~Hz}, 1 \mathrm{H} ; \mathrm{COOCH}), 7.28(\mathrm{~d}, J=9.3 \mathrm{~Hz}, 2 \mathrm{H} ; \mathrm{Ar}), 7.52(\mathrm{~d}, J=8.0 \mathrm{~Hz}, 2 \mathrm{H} ; \mathrm{Ar}) ;{ }^{19} \mathrm{~F}$ NMR (254 MHz, $\left.\mathrm{CDCl}_{3}\right): \delta=-144.82(\mathrm{~s}, 1 \mathrm{~F})$; MS: $m / z: 345\left(\mathrm{M}^{+}\right)$; HRMS Calcd. for $\mathrm{C}_{20} \mathrm{H}_{24} \mathrm{FNO}_{3}\left(\mathrm{M}^{+}\right): 345.1740$. Found: 345.1773 .

\section{Preparation of (S)-CFTA (5) by hydrolysis of $(S)$-CFTA ester of 18}

To a solution of $(S)$-CFTA ester of $\mathbf{1 8}(3.0 \mathrm{~g}, 8.6 \mathrm{mmol})$ in $\mathrm{THF} / \mathrm{H}_{2} \mathrm{O}(1: 1=50 \mathrm{~mL})$ was added $1 \mathrm{M} \mathrm{LiOH}(8.6 \mathrm{~mL}, 8.6$ $\mathrm{mmol}$ ) and the mixture was stirred at room temperature for $5 \mathrm{~min}$. After evaporation of THF at room temperature, the aqueous layer was washed with ether $(10 \mathrm{~mL}$ x 2), acidified with $10 \% \mathrm{HCl}(\mathrm{pH} 1)$ and extracted with AcOEt $(30 \mathrm{~mL} x$ $3)$. The combined organic layer was washed with brine and dried over $\mathrm{MgSO}_{4}$. Evaporation of the solvent at room temperature gave $(S)$-CFTA $(1.4 \mathrm{~g}, 87 \%)$ as a yellow oil.

\section{(S)-CFTA}

$[\alpha]_{\mathrm{D}}{ }^{25}-36.3\left(c=1.1\right.$ in $\left.\mathrm{CHCl}_{3}\right)$; IR (neat): $v=1756(\mathrm{C}=\mathrm{O}) \mathrm{cm}^{-1} ;{ }^{1} \mathrm{H}$ NMR $\left(270 \mathrm{MHz}, \mathrm{CDCl}_{3}\right): \delta=2.40\left(\mathrm{~s}, 3 \mathrm{H} ; \mathrm{CH}_{3}\right)$, $7.29(\mathrm{~d}, J=7.8 \mathrm{~Hz}, 2 \mathrm{H} ; \mathrm{Ar}), 7.55(\mathrm{~d}, J=8.1 \mathrm{~Hz}, 2 \mathrm{H} ; \mathrm{Ar}) ;{ }^{19} \mathrm{~F} \mathrm{NMR}\left(254 \mathrm{NHz}, \mathrm{CDCl}_{3}\right): \delta=-145.6(\mathrm{~s}, 1 \mathrm{~F}) ; \mathrm{MS}: m / z$ : $193\left(\mathrm{M}^{+}\right), 167\left(\mathrm{M}^{+}-\mathrm{CN}\right), 149\left(\mathrm{M}^{+}-\mathrm{CO}_{2}\right)$.

\section{$(\boldsymbol{R})$-CFTA}

$[\alpha]_{\mathrm{D}}{ }^{25}+36.8\left(c=1.2\right.$ in $\left.\mathrm{CHCl}_{3}\right) ; \mathrm{IR}($ neat $): \mathrm{v}=1764(\mathrm{C}=\mathrm{O}) \mathrm{cm}^{-1} ;{ }^{1} \mathrm{H}$ NMR $\left(270 \mathrm{MHz}, \mathrm{CDCl}_{3}\right): \delta=2.40\left(\mathrm{~s}, 3 \mathrm{H} ; \mathrm{CH}_{3}\right)$, $7.29(\mathrm{~d}, J=7.8 \mathrm{~Hz}, 2 \mathrm{H} ; \mathrm{Ar}), 7.55(\mathrm{~d}, J=8.1 \mathrm{~Hz}, 2 \mathrm{H} ; \mathrm{Ar}):{ }^{19} \mathrm{~F} \mathrm{NMR}\left(254 \mathrm{MHz}, \mathrm{CDCl}_{3}\right): \delta=-145.7(\mathrm{~s}, 1 \mathrm{~F}) ; \mathrm{MS}: m / z$ : $193\left(\mathrm{M}^{+}\right), 149\left(\mathrm{M}^{+}-\mathrm{CO}_{2}\right)$.

\section{Preparation of $(S)$ - and $(R)$-CFTA-Cl}

In a similar manner for preparation of $( \pm)$-CFTA-Cl, $(S)$ - and $(R)$-CFTA-Cl were obtained from $(S)$ - and $(R)$-CFTA, respectively.

\section{General Procedure for Preparation of CFTA and MTPA Esters of Secondary Alcohols.}

To a mixture of the alcohol 7-17 (10 mg, 0.04-0.09 mmol) in dry pyridine (2.0 eq.) and dry benzene ( $2 \mathrm{~mL})$ was added dropwise $(S)$-CFTA-Cl, $(R)$-CFTA-Cl, $(S)$-MTPA-Cl, or $(R)$-MTPA-Cl $(1.0$ eq.) and the mixture was stirred at room temperature for $1-2 \mathrm{~h}$. Water was added to the reaction mixture and benzene was evaporated. The resulting mixture was extracted with AcOEt $(5 \mathrm{~mL}$ x 3$)$. The combined organic layer was washed with brine, dried over $\mathrm{MgSO}_{4}$, and AcOEt was evaporated. The residual oil was purified by column chromatography on silica gel (eluent:

hexane/AcOEt $=4 / 1)$, or by recrystallization from $\mathrm{CHCl}_{3}$ to give $(S)$-CFTA ester, $(R)$-CFTA ester, $(R)$-MTPA ester, or $(S)$-MTPA ester, respectively, in $60-100 \%$ yields. In the case of $\mathbf{1 1}$ and 12, the corresponding MTPA derivatives could not be obtained.

Physical and Spectral Data for the (S)- and (R)-CFTA Esters of Benzhydrols 7-12.

(S)-(2-methylphenyl)phenylmethyl (S)-2-cyano-2-fluoro-2-(4-methylphenyl)acetate ((S)-CFTA ester of 7)

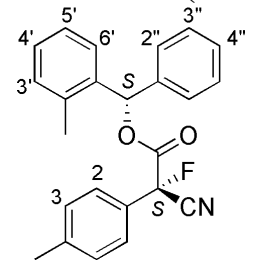

colorless oil; $[\alpha]_{\mathrm{D}}{ }^{29}+16.0\left(c=1.4\right.$ in $\left.\mathrm{CHCl}_{3}\right)$; IR (neat): $v=1772(\mathrm{C}=\mathrm{O}), 2254(\mathrm{CN}) \mathrm{cm}^{-1} ;{ }^{1} \mathrm{H}$ NMR $(500 \mathrm{MHz}$, $\left.\mathrm{CDCl}_{3}\right): \delta=2.28\left(\mathrm{~s}, 3 \mathrm{H} ;>\mathrm{CH}-\mathrm{Ar}-\mathrm{CH}_{3}\right), 2.39\left(\mathrm{~s}, 3 \mathrm{H} ;>\mathrm{CF}-\mathrm{Ar}-\mathrm{CH}_{3}\right), 7.04\left(\mathrm{~d}, J=7.7 \mathrm{~Hz}, 2 \mathrm{H} ; \mathrm{H}^{2 \prime}\right), 7.08\left(\mathrm{~s}, 1 \mathrm{H} ; \mathrm{H}^{\alpha}\right)$, $7.17\left(\mathrm{dd}, J=0.9,7.5 \mathrm{~Hz}, 1 \mathrm{H} ; \mathrm{H}^{3}\right), 7.20-7.24\left(\mathrm{~m}, 2 \mathrm{H} ; \mathrm{H}^{4^{\prime}}, \mathrm{H}^{4}\right), 7.22\left(\mathrm{~d}, J=9.0 \mathrm{~Hz}, 2 \mathrm{H} ; \mathrm{H}^{3}\right), 7.24(\mathrm{dd}, J=7.7,7.8$ $\left.\mathrm{Hz}, 2 \mathrm{H} ; \mathrm{H}^{3 "}\right), 7.25\left(\mathrm{dd}, J=7.3,8.6 \mathrm{~Hz}, 1 \mathrm{H} ; \mathrm{H}^{5}\right), 7.35\left(\mathrm{dd}, J=1.5,8.6 \mathrm{~Hz}, 1 \mathrm{H} ; \mathrm{H}^{6}\right), 7.44\left(\mathrm{~d}, J=9.0 \mathrm{~Hz}, 2 \mathrm{H}, \mathrm{H}^{2}\right)$; ${ }^{19} \mathrm{~F} \mathrm{NMR}\left(254 \mathrm{MHz}, \mathrm{CDCl}_{3}\right): \delta=-144.85$ (s, $\left.1 \mathrm{~F}\right)$; MS: $m / z$ : $373\left(\mathrm{M}^{+}\right)$; HRMS Calcd. for $\mathrm{C}_{24} \mathrm{H}_{21} \mathrm{FNO}_{2}\left(\mathrm{M}^{+}+1\right)$ : 374.1556. Found: 374.1524.

$(S)$-(2-methylphenyl)phenylmethyl $(R)$-2-cyano-2-fluoro-2-(4-methylphenyl)acetate $((R)$-CFTA ester of 7) 


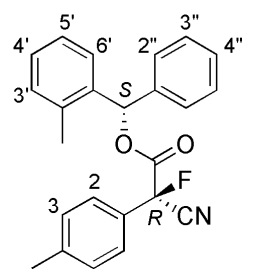

colorless oil; $[\alpha]_{\mathrm{D}}{ }^{28}-2.9\left(c=0.6\right.$ in $\left.\mathrm{CHCl}_{3}\right)$; IR (neat): $v=1771(\mathrm{C}=\mathrm{O}), 2253(\mathrm{CN}) \mathrm{cm}^{-1} ;{ }^{1} \mathrm{H} \mathrm{NMR}(500 \mathrm{MHz}$, $\left.\mathrm{CDCl}_{3}\right): \delta=2.16\left(\mathrm{~s}, 3 \mathrm{H} ;>\mathrm{CH}-\mathrm{Ar}-\mathrm{CH}_{3}\right), 2.39\left(\mathrm{~s}, 3 \mathrm{H}:>\mathrm{CF}-\mathrm{Ar}-\mathrm{CH}_{3}\right), 7.03\left(\mathrm{~d}, J=7.5 \mathrm{~Hz}, 1 \mathrm{H} ; \mathrm{H}^{6}\right), 7.07(\mathrm{dd}, J=7.5$, $\left.7.8 \mathrm{~Hz}, 1 \mathrm{H} ; \mathrm{H}^{5}\right), 7.07\left(\mathrm{~s}, 1 \mathrm{H} ; \mathrm{H}^{\alpha}\right), 7.10\left(\mathrm{~d}, J=7.7 \mathrm{~Hz}, 1 \mathrm{H} ; \mathrm{H}^{3}\right), 7.19\left(\mathrm{dd}, J=7.7,7.8 \mathrm{~Hz}, 1 \mathrm{H} ; \mathrm{H}^{4}\right), 7.22(\mathrm{~d}, J=$ $\left.7.7 \mathrm{~Hz}, 2 \mathrm{H} ; \mathrm{H}^{3}\right), 7.25\left(\mathrm{dd}, J=2.0,7.6 \mathrm{~Hz}, 2 \mathrm{H} ; \mathrm{H}^{2 ”}\right), 7.30-7.35\left(\mathrm{~m}, 3 \mathrm{H} ; \mathrm{H}^{3,}, \mathrm{H}^{4 "}\right), 7.43\left(\mathrm{~d}, J=7.7 \mathrm{~Hz}, 2 \mathrm{H} ; \mathrm{H}^{2}\right) ;{ }^{19} \mathrm{~F}$ NMR (254.19 MHz, $\left.\mathrm{CDCl}_{3}\right) \delta:=-143.68$ (s, 1F); MS: $m / z: 373\left(\mathrm{M}^{+}\right)$; HRMS Calcd. for $\mathrm{C}_{24} \mathrm{H}_{20} \mathrm{FNO}_{2}\left(\mathrm{M}^{+}\right)$: 373.1602. Found: 373.1442 .

(S)-(2-bromophenyl)phenylmethyl (S)-2-cyano-2-fluoro-2-(4-methylphenyl)acetate ((S)-CFTA ester of 8)

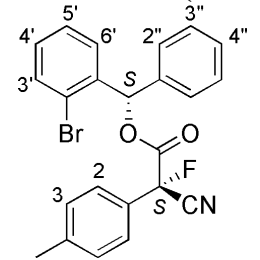

yellow oil; $[\alpha]_{\mathrm{D}}{ }^{30}+17.3\left(c=0.5\right.$ in $\left.\mathrm{CHCl}_{3}\right)$; IR (neat): $\mathrm{v}=1774(\mathrm{C}=\mathrm{O}), 2254(\mathrm{CN}) \mathrm{cm}^{-1} ;{ }^{1} \mathrm{H} \mathrm{NMR}(500 \mathrm{MHz}$, $\left.\mathrm{CDCl}_{3}\right): \delta=2.40\left(\mathrm{~s}, 3 \mathrm{H} ; \mathrm{CH}_{3}\right), 7.13\left(\mathrm{dd}, J=1.5,7.3 \mathrm{~Hz}, 2 \mathrm{H} ; \mathrm{H}^{2 "}\right), 7.21\left(\mathrm{ddd}, J=1.8,7.3,7.8 \mathrm{~Hz}, 1 \mathrm{H} ; \mathrm{H}^{4}\right), 7.23$ $\left(\mathrm{d}, J=7.7 \mathrm{~Hz}, 2 \mathrm{H} ; \mathrm{H}^{3}\right), 7.24\left(\mathrm{~s}, 1 \mathrm{H} ; \mathrm{H}^{\alpha}\right), 7.21-7.28\left(\mathrm{~m}, 3 \mathrm{H} ; \mathrm{H}^{3}, \mathrm{H}^{4}\right), 7.34\left(\mathrm{ddd}, J=1.2,7.3,7.4 \mathrm{~Hz}, 1 \mathrm{H} ; \mathrm{H}^{5}\right)$, $7.40\left(\mathrm{dd}, J=1.8,7.4 \mathrm{~Hz}, 1 \mathrm{H} ; \mathrm{H}^{6}\right), 7.44\left(\mathrm{~d}, J=7.7 \mathrm{~Hz}, 2 \mathrm{H} ; \mathrm{H}^{2}\right), 7.58\left(\mathrm{dd}, J=1.2,7.8 \mathrm{~Hz}, 1 \mathrm{H} ; \mathrm{H}^{3}\right) ;{ }^{19} \mathrm{~F} \mathrm{NMR}(254$ $\left.\mathrm{MHz}, \mathrm{CDCl}_{3}\right): \delta=-144.70$ (s, 1F); MS: $m / z: 437,439\left(\mathrm{M}^{+}\right)$; HRMS Calcd. for $\mathrm{C}_{23} \mathrm{H}_{17}{ }^{79} \mathrm{BrFNO}_{2}\left(\mathrm{M}^{+}\right)$: 437.0426. Found: 437.0383. Calcd. for $\mathrm{C}_{23} \mathrm{H}_{17}{ }^{81} \mathrm{BrFNO}_{2}\left(\mathrm{M}^{+}\right)$: 439.0407. Found: 439.0388.

(S)-(2-bromophenyl)phenylmethyl (R)-2-cyano-2-fluoro-2-(4-methylphenyl)acetate $((R)$-CFTA ester of 8)

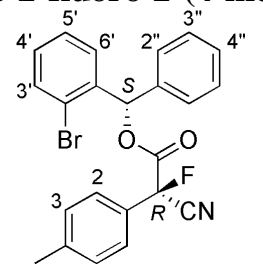

colorless oil; $[\alpha]_{\mathrm{D}}{ }^{29}+1.0\left(c=1.8\right.$ in $\left.\mathrm{CHCl}_{3}\right)$; IR (neat): $\mathrm{v}=1775(\mathrm{C}=\mathrm{O}), 2255(\mathrm{CN}) \mathrm{cm}^{-1} ;{ }^{1} \mathrm{H}$ NMR $(500 \mathrm{MHz}$, $\left.\mathrm{CDCl}_{3}\right): \delta=2.40\left(\mathrm{~s}, 3 \mathrm{H} ; \mathrm{CH}_{3}\right), 7.07\left(\mathrm{dd}, J=2.2,6.7 \mathrm{~Hz}, 1 \mathrm{H} ; \mathrm{H}^{6^{\prime}}\right), 7.14\left(\mathrm{ddd}, J=2.0,6.3,6.7 \mathrm{~Hz}, 1 \mathrm{H} ; \mathrm{H}^{5}\right), 7.16$ $\left(\mathrm{ddd}, J=2.2,6.3,6.8 \mathrm{~Hz}, 1 \mathrm{H} ; \mathrm{H}^{4}\right), 7.22\left(\mathrm{~s}, 1 \mathrm{H} ; \mathrm{H}^{\alpha}\right), 7.24\left(\mathrm{~d}, J=7.9 \mathrm{~Hz}, 2 \mathrm{H} ; \mathrm{H}^{3}\right), 7.32-7.38\left(\mathrm{~m}, 5 \mathrm{H} ; \mathrm{H}^{2}, \mathrm{H}^{3 "}\right.$, $\left.\mathrm{H}^{4 \prime \prime}\right), 7.47\left(\mathrm{~d}, J=7.9 \mathrm{~Hz}, 2 \mathrm{H} ; \mathrm{H}^{2}\right), 7.52\left(\mathrm{dd}, J=2.0,6.8 \mathrm{~Hz}, 2 \mathrm{H} ; \mathrm{H}^{3}\right) ;{ }^{\prime}{ }^{1} \mathrm{~F}$ NMR $\left(254 \mathrm{MHz}, \mathrm{CDCl}_{3}\right): \delta=-144.07$ (s, 1F); MS: $m / z$ : 437, $439\left(\mathrm{M}^{+}\right)$; HRMS Calcd. for $\mathrm{C}_{23} \mathrm{H}_{17}{ }^{79} \mathrm{BrFNO}_{2}\left(\mathrm{M}^{+}\right)$: 437.0481. Found: 437.0421. Calcd. for $\mathrm{C}_{23} \mathrm{H}_{17}{ }^{81} \mathrm{BrFNO}_{2}\left(\mathrm{M}^{+}\right): 439.0361$. Found: 439.0411 .

(S)-(2-chlorophenyl)phenylmethyl (S)-2-cyano-2-fluoro-2-(4-methylphenyl)acetate ((S)-CFTA ester of 9)

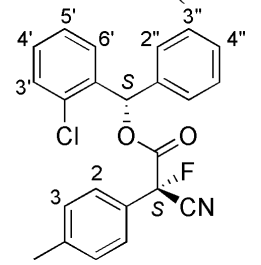

colorless oil; $[\alpha]_{\mathrm{D}}{ }^{29}+26.3\left(c=0.3\right.$ in $\left.\mathrm{CHCl}_{3}\right)$; IR (neat): $1775(\mathrm{C}=\mathrm{O}), 2255(\mathrm{CN}) \mathrm{cm}^{-1} ;{ }^{1} \mathrm{H}$ NMR $(500 \mathrm{MHz}$, $\left.\mathrm{CDCl}_{3}\right): \delta=2.39\left(\mathrm{~s}, 3 \mathrm{H} ; \mathrm{CH}_{3}\right), 7.13\left(\mathrm{~d}, J=7.5 \mathrm{~Hz}, 2 \mathrm{H} ; \mathrm{H}^{2 \prime}\right), 7.23\left(\mathrm{~d}, J=8.0 \mathrm{~Hz}, 2 \mathrm{H} ; \mathrm{H}^{3}\right), 7.22-7.25\left(\mathrm{~m}, 2 \mathrm{H} ; \mathrm{H}^{3 ”}\right)$, 7.25-7.27 (m, 1H; $\left.\mathrm{H}^{4^{\prime \prime}}, \mathrm{H}^{\alpha}\right), 7.27-7.30\left(\mathrm{~m}, 2 \mathrm{H} ; \mathrm{H}^{4}, \mathrm{H}^{5^{\prime}}\right), 7.38-7.40\left(\mathrm{~m}, 1 \mathrm{H} ; \mathrm{H}^{6^{\prime}}\right), 7.40-7.43\left(\mathrm{~m}, 1 \mathrm{H} ; \mathrm{H}^{3}\right), 7.44(\mathrm{~d}$, $\left.J=8.0 \mathrm{~Hz}, 2 \mathrm{H} ; \mathrm{H}^{2}\right) ;{ }^{19} \mathrm{~F}$ NMR $\left(254 \mathrm{MHz}, \mathrm{CDCl}_{3}\right): \delta=-144.54$ (s, 1F); MS: $m / z: 393$, $395\left(\mathrm{M}^{+}\right)$; HRMS Calcd. for $\mathrm{C}_{23} \mathrm{H}_{17}{ }^{35} \mathrm{ClFNO}_{2}\left(\mathrm{M}^{+}\right)$: 393.0926. Found: 393.0932. Calcd. for $\mathrm{C}_{23} \mathrm{H}_{17}{ }^{37} \mathrm{ClFNO}_{2}\left(\mathrm{M}^{+}\right)$: 395.0866. Found: 395.0902 . 
(S)-(2-chlorophenyl)phenylmethyl $(\boldsymbol{R})$-2-cyano-2-fluoro-2-(4-methylphenyl)acetate $((R)-C F T A$ ester of 9)

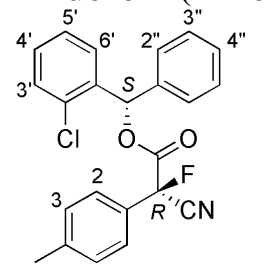

colorless oil; $[\alpha]_{\mathrm{D}}{ }^{29}+11.1\left(c=0.1\right.$ in $\left.\mathrm{CHCl}_{3}\right)$; IR (neat): $\mathrm{v}=1775(\mathrm{C}=\mathrm{O}), 2254(\mathrm{CN}) \mathrm{cm}^{-1} ;{ }^{1} \mathrm{H} \mathrm{NMR}(500 \mathrm{MHz}$, $\left.\mathrm{CDCl}_{3}\right): \delta=2.40\left(\mathrm{~d}, J=1.1 \mathrm{~Hz}, 3 \mathrm{H} ; \mathrm{CH}_{3}\right), 7.09\left(\mathrm{dd}, J=1.8,7.7 \mathrm{~Hz}, 1 \mathrm{H} ; \mathrm{H}^{6}\right), 7.13(\mathrm{ddd}, J=1.3,7.6,7.7 \mathrm{~Hz}, 1 \mathrm{H}$; $\mathrm{H}^{5}$ ), $7.22\left(\mathrm{ddd}, J=1.8,7.6,7.6 \mathrm{~Hz}, 1 \mathrm{H} ; \mathrm{H}^{4}\right), 7.24\left(\mathrm{~d}, J=7.8 \mathrm{~Hz}, 2 \mathrm{H} ; \mathrm{H}^{3}\right), 7.26\left(\mathrm{~s}, 1 \mathrm{H} ; \mathrm{H}^{\alpha}\right), 7.32(\mathrm{dd}, J=1.3,7.6$ $\left.\mathrm{Hz}, 1 \mathrm{H} ; \mathrm{H}^{3}\right), 7.32-7.36\left(\mathrm{~m}, 5 \mathrm{H} ; \mathrm{H}^{2}\right.$ ", $\mathrm{H}^{3}$ ", $\mathrm{H}^{4}$ ") $7.47\left(\mathrm{~d}, J=7.8 \mathrm{~Hz}, 2 \mathrm{H} ; \mathrm{H}^{2}\right) ;{ }^{19} \mathrm{~F}$ NMR $\left(254 \mathrm{MHz} \mathrm{CDCl}_{3}\right): \delta=$ -144.03 (s, 1F); MS: $m / z$ : 393, $395\left(\mathrm{M}^{+}\right)$; HRMS Calcd. for $\mathrm{C}_{23} \mathrm{H}_{17}{ }^{35} \mathrm{ClFNO}_{2}\left(\mathrm{M}^{+}\right)$: 393.0805. Found: 393.0945. Calcd. for $\mathrm{C}_{23} \mathrm{H}_{17}{ }^{37} \mathrm{ClFNO}^{2}\left(\mathrm{M}^{+}\right)$: 395.0866. Found: 395.0906.

$(S)$-(2-fluorophenyl)phenylmethyl $(S)$-2-cyano-2-fluoro-2-(4-methylphenyl)acetate $((S)$-CFTA ester of 10)

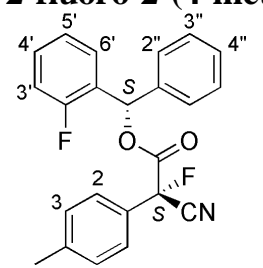

colorless oil; $[\alpha]_{\mathrm{D}}{ }^{30}+13.2\left(c=0.5\right.$ in $\left.\mathrm{CHCl}_{3}\right)$; IR (neat): $v=1776(\mathrm{C}=\mathrm{O}), 2255(\mathrm{CN}) \mathrm{cm}^{-1} ;{ }^{1} \mathrm{H} \mathrm{NMR}(500 \mathrm{MHz}$, $\left.\mathrm{CDCl}_{3}\right): \delta=2.40\left(\mathrm{~s}, 3 \mathrm{H} ; \mathrm{CH}_{3}\right), 7.07\left(\mathrm{dd}, J=8.5,9.2 \mathrm{~Hz}, 1 \mathrm{H} ; \mathrm{H}^{3}\right), 7.13\left(\mathrm{dd}, J=1.3,8.7 \mathrm{~Hz}, 2 \mathrm{H} ; \mathrm{H}^{2 \prime}\right), 7.12-7.17$ $\left(\mathrm{m}, 1 \mathrm{H} ; \mathrm{H}^{5}\right), 7.18\left(\mathrm{~s}, 1 \mathrm{H} ; \mathrm{H}^{\alpha}\right), 7.24\left(\mathrm{~d}, J=7.8 \mathrm{~Hz}, 2 \mathrm{H} ; \mathrm{H}^{3}\right), 7.23-7.27\left(\mathrm{~m}, 3 \mathrm{H} ; \mathrm{H}^{3 "}, \mathrm{H}^{4}\right.$ ) $), 7.33(\mathrm{ddd}, J=1.8,7.8$, $\left.8.5 \mathrm{~Hz}, 1 \mathrm{H} ; \mathrm{H}^{4}\right), 7.35\left(\mathrm{dd}, J=1.8,8.1 \mathrm{~Hz}, 1 \mathrm{H} ; \mathrm{H}^{6}\right), 7.46\left(\mathrm{~d}, J=7.8 \mathrm{~Hz}, 2 \mathrm{H} ; \mathrm{H}^{2}\right) ;{ }^{19} \mathrm{~F} \mathrm{NMR}^{\prime}\left(254 \mathrm{MHz}, \mathrm{CDCl}_{3}\right): \delta$ $=-117.33(\mathrm{~m}, 1 \mathrm{~F}),-144.37(\mathrm{~s}, 1 \mathrm{~F})$; MS: $m / z: 377\left(\mathrm{M}^{+}\right)$; HRMS Calcd. for $\mathrm{C}_{23} \mathrm{H}_{17} \mathrm{~F}_{2} \mathrm{NO}_{2}\left(\mathrm{M}^{+}\right)$: 377.1227 . Found: 377.1232 .

(S)-(2-fluorophenyl)phenylmethyl $(\boldsymbol{R})$-2-cyano-2-fluoro-2-(4-methylphenyl)acetate $((R)$-CFTA ester of 10)

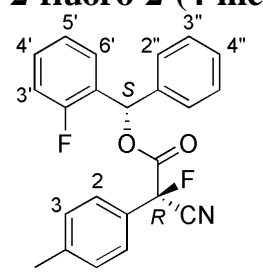

colorless oil; $[\alpha]_{\mathrm{D}}{ }^{29}+0.4\left(c=0.2\right.$ in $\left.\mathrm{CHCl}_{3}\right)$; IR (neat): $\mathrm{v}=1776(\mathrm{C}=\mathrm{O}), 2257(\mathrm{CN}) \mathrm{cm}^{-1} ;{ }^{1} \mathrm{H} \mathrm{NMR}(500 \mathrm{MHz}$, $\left.\mathrm{CDCl}_{3}\right): \delta=2.40\left(\mathrm{~s}, 3 \mathrm{H} ; \mathrm{CH}_{3}\right), 6.97-7.02\left(\mathrm{~m}, 1 \mathrm{H} ; \mathrm{H}^{3}\right), 7.01\left(\mathrm{~d}, J=7.4 \mathrm{~Hz}, 1 \mathrm{H} ; \mathrm{H}^{6}\right), 7.04(\mathrm{ddd}, J=1.9,7.4,7.5$ $\left.\mathrm{Hz}, 1 \mathrm{H} ; \mathrm{H}^{5}\right), 7.18\left(\mathrm{~s}, 1 \mathrm{H} ; \mathrm{H}^{\alpha}\right), 7.24\left(\mathrm{~d}, J=7.8 \mathrm{~Hz}, 2 \mathrm{H} ; \mathrm{H}^{3}\right), 7.24-7.28\left(\mathrm{~m}, 1 \mathrm{H} ; \mathrm{H}^{4}\right), 7.32-7.38\left(\mathrm{~m}, 5 \mathrm{H} ; \mathrm{H}^{2 ”}, \mathrm{H}^{3 "}\right.$, $\mathrm{H}^{4}$ ) $), 7.48\left(\mathrm{~d}, J=7.8 \mathrm{~Hz}, 2 \mathrm{H} ; \mathrm{H}^{2}\right) ;{ }^{19} \mathrm{~F} \mathrm{NMR}(254 \mathrm{MHz}, \mathrm{CDCl} 3): \delta=-117.36(\mathrm{ddd}, 5.6,5.6,10.2 \mathrm{~Hz}, 1 \mathrm{~F})$, -144.29 (s, 1F); MS: m/z: $377\left(\mathrm{M}^{+}\right)$; HRMS Calcd. for $\mathrm{C}_{23} \mathrm{H}_{18} \mathrm{~F}_{2} \mathrm{NO}_{2}\left(\mathrm{M}^{+}+1\right)$ : 378.1305. Found: 378.1339 .

(S)-(4-methylphenyl)phenylmethyl (S)-2-cyano-2-fluoro-2-(4-methylphenyl)acetate ((S)-CFTA ester of 11)

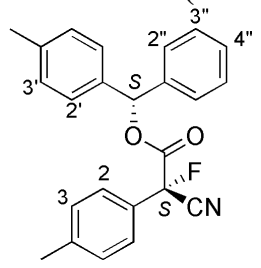

colorless oil; $[\alpha]_{\mathrm{D}}{ }^{29}-0.1\left(c=1.9\right.$ in $\left.\mathrm{CHCl}_{3}\right)$; IR (neat): $\mathrm{v}=1771(\mathrm{C}=\mathrm{O}), 2254(\mathrm{CN}) \mathrm{cm}^{-1} ;{ }^{1} \mathrm{H} \mathrm{NMR}(500 \mathrm{MHz}$ $\left.\mathrm{CDCl}_{3}\right): \delta=2.33\left(\mathrm{~s}, 3 \mathrm{H} ;>\mathrm{CH}-\mathrm{Ar}-\mathrm{CH}_{3}\right), 2.39\left(\mathrm{~s}, 3 \mathrm{H} ;>\mathrm{CF}-\mathrm{Ar}-\mathrm{CH}_{3}\right), 6.88\left(\mathrm{~s}, 1 \mathrm{H} ; \mathrm{H}^{\alpha}\right), 7.09(\mathrm{dd}, J=2.0,8.0 \mathrm{~Hz}$, $\left.2 \mathrm{H} ; \mathrm{H}^{2}\right), 7.15\left(\mathrm{~d}, J=8.1 \mathrm{~Hz}, 2 \mathrm{H} ; \mathrm{H}^{3}\right), 7.19\left(\mathrm{~d}, J=8.1 \mathrm{~Hz}, 2 \mathrm{H} ; \mathrm{H}^{2}\right), 7.22\left(\mathrm{~d}, J=8.3 \mathrm{~Hz}, 2 \mathrm{H} ; \mathrm{H}^{3}\right), 7.23(\mathrm{dd}, J=$ 8.0, 8.0 Hz, 2H; $\left.\mathrm{H}^{3 "}\right), 7.26\left(\mathrm{dd}, J=8.0,8.0 \mathrm{~Hz}, 1 \mathrm{H} ; \mathrm{H}^{4 "}\right), 7.45\left(\mathrm{~d}, J=8.3 \mathrm{~Hz}, 2 \mathrm{H} ; \mathrm{H}^{2}\right) ;{ }^{19} \mathrm{~F} \mathrm{NMR}(254 \mathrm{MHz}$, 
$\left.\mathrm{CDCl}_{3}\right): \delta=-144.28(\mathrm{~s}, 1 \mathrm{~F})$; MS: $m / z: 373\left(\mathrm{M}^{+}\right)$; HRMS Calcd. for $\mathrm{C}_{24} \mathrm{H}_{20} \mathrm{FNO}_{2}\left(\mathrm{M}^{+}\right): 373.1479$. Found: 373.1516 .

$(S)$-(4-methylphenyl)phenylmethyl $(R)$-2-cyano-2-fluoro-2-(4-methylphenyl)acetate $((R)$-CFTA ester of 11)

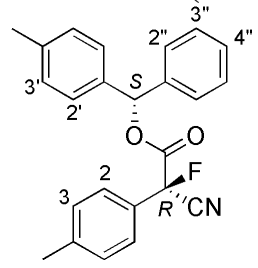

colorless oil; $[\alpha]_{\mathrm{D}}{ }^{28}-10.6\left(c=1.7\right.$ in $\left.\mathrm{CHCl}_{3}\right)$; IR (neat): $\mathrm{v}=1771(\mathrm{C}=\mathrm{O}), 2254(\mathrm{CN}) \mathrm{cm}^{-1} ;{ }^{1} \mathrm{H} \mathrm{NMR}(500 \mathrm{MHz}$, $\left.\mathrm{CDCl}_{3}\right): \delta=2.30\left(\mathrm{~s}, 3 \mathrm{H} ;>\mathrm{CH}-\mathrm{Ar}-\mathrm{CH}_{3}\right), 2.39\left(\mathrm{~s}, 3 \mathrm{H} ;>\mathrm{CF}-\mathrm{Ar}-\mathrm{CH}_{3}\right), 6.88\left(\mathrm{~s}, 1 \mathrm{H} ; \mathrm{H}^{\alpha}\right), 6.99\left(\mathrm{~d}, J=8.2 \mathrm{~Hz}, 2 \mathrm{H} ; \mathrm{H}^{2}\right)$, $7.05\left(\mathrm{~d}, J=8.2 \mathrm{~Hz}, 2 \mathrm{H} ; \mathrm{H}^{3}\right), 7.22\left(\mathrm{~d}, J=8.0 \mathrm{~Hz}, 2 \mathrm{H} ; \mathrm{H}^{3}\right), 7.23\left(\mathrm{~d}, J=6.9 \mathrm{~Hz}, 2 \mathrm{H} ; \mathrm{H}^{2}\right), 7.28-7.32\left(\mathrm{~m}, 1 \mathrm{H} ; \mathrm{H}^{4}\right)$, $7.33\left(\mathrm{dd}, J=6.6,6.9 \mathrm{~Hz}, 2 \mathrm{H} ; \mathrm{H}^{3}\right), 7.43\left(\mathrm{~d}, J=8.0 \mathrm{~Hz}, 2 \mathrm{H} ; \mathrm{H}^{2}\right) ;{ }^{19} \mathrm{~F} \mathrm{NMR}\left(254 \mathrm{MHz}, \mathrm{CDCl}_{3}\right): \delta=-144.42(\mathrm{~s}$, 1F); MS: $m / z: 373\left(\mathrm{M}^{+}\right)$; HRMS Calcd. for $\mathrm{C}_{24} \mathrm{H}_{20} \mathrm{FNO}_{2}\left(\mathrm{M}^{+}\right)$: 373.1478 . Found: 373.1454 .

(S)-(4-trifluoromethylphenyl)phenylmethyl (S)-2-cyano-2-fluoro-2-(4-methylphenyl)acetate $((S)$-CFTA ester of 12)

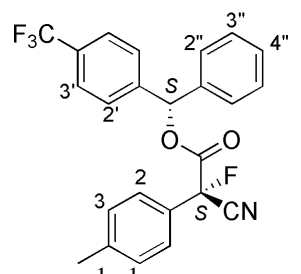

yellow oil; IR (neat): $v=1775(\mathrm{C}=\mathrm{O}), 2254(\mathrm{CN}) \mathrm{cm}^{-1} ;{ }^{1} \mathrm{H}$ NMR $\left(500 \mathrm{MHz}, \mathrm{CDCl}_{3}\right): \delta=2.39\left(\mathrm{~s}, 3 \mathrm{H} ; \mathrm{CH}_{3}\right), 6.93$ $\left(\mathrm{s}, 1 \mathrm{H} ; \mathrm{H}^{\alpha}\right), 7.10\left(\mathrm{dd}, J=1.5,7.9 \mathrm{~Hz}, 2 \mathrm{H} ; \mathrm{H}^{2 " \prime}\right), 7.23\left(\mathrm{~d}, J=8.6 \mathrm{~Hz}, 2 \mathrm{H} ; \mathrm{H}^{3}\right), 7.23-7.30\left(\mathrm{~m}, 3 \mathrm{H} ; \mathrm{H}^{3, "}, \mathrm{H}^{4 \prime}\right), 7.43(\mathrm{~d}$, $\left.J=8.6 \mathrm{~Hz}, 2 \mathrm{H} ; \mathrm{H}^{2}\right), 7.43\left(\mathrm{~d}, J=8.4 \mathrm{~Hz}, 2 \mathrm{H} ; \mathrm{H}^{2}\right), 7.61\left(\mathrm{~d}, J=8.4 \mathrm{~Hz}, 2 \mathrm{H} ; \mathrm{H}^{3}\right) ;{ }^{\prime} \mathrm{F} \mathrm{NMR}^{2}\left(254 \mathrm{MHz}, \mathrm{CDCl}_{3}\right): \delta=$ -63.29 (s, 3F), -144.79 (s, 1F); MS: $m / z: 427\left(\mathrm{M}^{+}\right)$; HRMS Calcd. for $\mathrm{C}_{24} \mathrm{H}_{17} \mathrm{~F}_{4} \mathrm{NO}_{2}\left(\mathrm{M}^{+}\right)$: 427.1195. Found: 427.1186 .

(S)-(4-trifluoromethylphenyl)phenylmethyl $(R)$-2-cyano-2-fluoro-2-(4-methylphenyl)acetate $((R)$-CFTA ester of 12)

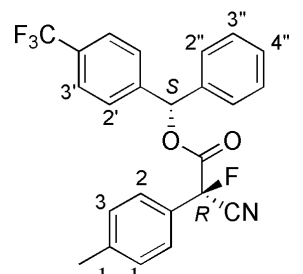

yellow oil; IR (neat): $v=1775(\mathrm{C}=\mathrm{O}), 2254(\mathrm{CN}) \mathrm{cm}^{-1} ;{ }^{1} \mathrm{H}$ NMR $\left(500 \mathrm{MHz}, \mathrm{CDCl}_{3}\right): \delta=2.40\left(\mathrm{~s}, 3 \mathrm{H} ; \mathrm{CH}_{3}\right), 6.93$ $\left(\mathrm{s}, 1 \mathrm{H} ; \mathrm{H}^{\alpha}\right), 7.20\left(\mathrm{~d}, J=8.2 \mathrm{~Hz}, 2 \mathrm{H} ; \mathrm{H}^{2}\right), 7.23\left(\mathrm{~d}, J=7.6 \mathrm{~Hz}, 2 \mathrm{H} ; \mathrm{H}^{3}\right), 7.29\left(\mathrm{dd}, J=2.7,9.2 \mathrm{~Hz}, 1 \mathrm{H} ; \mathrm{H}^{2 "}\right)$, $7.34-7.38\left(\mathrm{~m}, 3 \mathrm{H} ; \mathrm{H}^{3, "}, \mathrm{H}^{4}\right), 7.44\left(\mathrm{~d}, J=7.6 \mathrm{~Hz}, 2 \mathrm{H} ; \mathrm{H}^{2}\right), 7.49\left(\mathrm{~d}, J=8.2 \mathrm{~Hz}, 2 \mathrm{H} ; \mathrm{H}^{3{ }^{\prime}}\right) ;{ }^{19} \mathrm{~F}$ NMR $(254 \mathrm{MHz}$, $\left.\mathrm{CDCl}_{3}\right): \delta=-63.32(\mathrm{~s}, 3 \mathrm{~F}),-144.54$ (s, 1F); MS: $m / z: 427\left(\mathrm{M}^{+}\right)$; HRMS Calcd. for $\mathrm{C}_{24} \mathrm{H}_{17} \mathrm{~F}_{4} \mathrm{NO}_{2}\left(\mathrm{M}^{+}\right): 427.1195$. Found: 427.1206 .

Physical and Spectral Data for the (S)- and (R) -MTPA Esters of Benzhydrols 7-10.

(S)-(2-methylphenyl)phenylmethyl (S)-3,3,3-trifluoro-2-methoxy-2-phenylpropanoate ((S)-MTPA ester of 7)

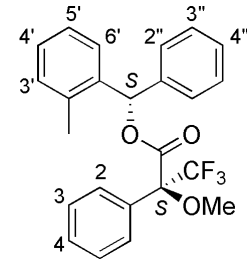


colorless oil; $[\alpha]_{\mathrm{D}}{ }^{26}-24.3\left(c=0.2\right.$ in $\left.\mathrm{CHCl}_{3}\right)$; IR $\left(\mathrm{CHCl}_{3}\right): v=1747(\mathrm{C}=\mathrm{O}) \mathrm{cm}^{-1} ;{ }^{1} \mathrm{H} \mathrm{NMR}\left(500 \mathrm{MHz}, \mathrm{CDCl}_{3}\right): \delta$ $=2.31\left(\mathrm{~s}, 3 \mathrm{H} ; \mathrm{CH}_{3}\right), 3.47\left(\mathrm{~s}, 3 \mathrm{H} ; \mathrm{OCH}_{3}\right), 7.17\left(\mathrm{~d}, J=6.8 \mathrm{~Hz}, 1 \mathrm{H} ; \mathrm{H}^{3}\right), 7.17-7.20(\mathrm{~m}, 2 \mathrm{H} ; \mathrm{Ar}), 7.22(\mathrm{ddd}, J=1.9$, $\left.6.8,7.3 \mathrm{~Hz}, 1 \mathrm{H} ; \mathrm{H}^{4}\right), 7.24\left(\mathrm{dd}, J=7.0,7.3 \mathrm{~Hz}, 1 \mathrm{H} ; \mathrm{H}^{5}\right), 7.24-7.28(\mathrm{~m}, 3 \mathrm{H} ; \mathrm{Ar}), 7.26\left(\mathrm{~s}, 1 \mathrm{H} ; \mathrm{H}^{\alpha}\right), 7.30(\mathrm{dd}, J=$ $\left.7.0,8.5 \mathrm{~Hz}, 2 \mathrm{H} ; \mathrm{H}^{3 \prime}\right), 7.37\left(\mathrm{~d}, J=8.5 \mathrm{~Hz}, 2 \mathrm{H} ; \mathrm{H}^{2 "}\right), 7.37\left(\mathrm{dd}, J=7.0,7.0 \mathrm{~Hz}, 1 \mathrm{H} ; \mathrm{H}^{4 \prime}\right), 7.42(\mathrm{dd}, J=1.9,7.0 \mathrm{~Hz}$, $\left.1 \mathrm{H} ; \mathrm{H}^{6}\right) ;{ }^{6} \mathrm{~F}$ NMR $\left(254 \mathrm{MHz}, \mathrm{CDCl}_{3}\right): \delta=-71.79(\mathrm{~s}, 3 \mathrm{~F})$; MS: $m / z: 414\left(\mathrm{M}^{+}\right)$; HRMS Calcd. for $\mathrm{C}_{24} \mathrm{H}_{21} \mathrm{~F}_{3} \mathrm{O}_{3}$ $\left(\mathrm{M}^{+}\right)$: 414.1443. Found: 414.1491.

(S)-(2-methylphenyl)phenylmethyl (R)-3,3,3-trifluoro-2-methoxy-2-phenylpropanoate $((R)$-MTPA ester of 7)

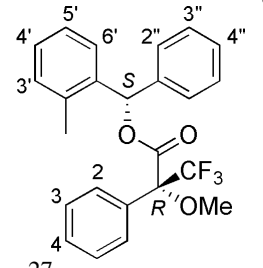

colorless solid (from $\mathrm{CHCl}_{3}$ ); M.p. $38-40{ }^{\circ} \mathrm{C} ;[\alpha]_{\mathrm{D}}{ }^{27}+33.4\left(c=2.7\right.$ in $\left.\mathrm{CHCl}_{3}\right)$; IR $(\mathrm{KBr}): v=1756(\mathrm{C}=\mathrm{O}) \mathrm{cm}^{-1}$; ${ }^{1} \mathrm{H}$ NMR $\left(500 \mathrm{MHz}, \mathrm{CDCl}_{3}\right): \delta=2.24\left(\mathrm{~s}, 3 \mathrm{H} ; \mathrm{CH}_{3}\right), 3.50\left(\mathrm{~s}, 3 \mathrm{H} ; \mathrm{OCH}_{3}\right), 7.13\left(\mathrm{~d}, J=7.1 \mathrm{~Hz}, 1 \mathrm{H} ; \mathrm{H}^{3}\right), 7.14(\mathrm{dd}, J$ $\left.=6.9,7.1 \mathrm{~Hz}, 1 \mathrm{H} ; \mathrm{H}^{4}\right), 7.21\left(\mathrm{dd}, J=6.9,8.1 \mathrm{~Hz}, 1 \mathrm{H} ; \mathrm{H}^{5^{\prime}}\right), 7.23\left(\mathrm{~s}, 1 \mathrm{H} ; \mathrm{H}^{\alpha}\right), 7.24\left(\mathrm{~d}, J=8.1 \mathrm{~Hz}, 1 \mathrm{H} ; \mathrm{H}^{6}\right), 7.28(\mathrm{dd}$, $\left.J=8.1,8.5 \mathrm{~Hz}, 2 \mathrm{H} ; \mathrm{H}^{3 \prime}\right), 7.27-7.32\left(\mathrm{~m}, 5 \mathrm{H}, \mathrm{H}^{2}, \mathrm{H}^{3}, \mathrm{H}^{4}\right), 7.34$ (d, J = 8.1 Hz, 2H; $\left.\mathrm{H}^{2 \prime}\right), 7.35-7.39$ (m, $\left.1 \mathrm{H} ; \mathrm{H}^{4 "}\right)$; ${ }^{19} \mathrm{~F}$ NMR $\left(254 \mathrm{MHz}, \mathrm{CDCl}_{3}\right): \delta=-71.86(\mathrm{~s}, 3 \mathrm{~F})$; MS: $\mathrm{m} / z$ : $414\left(\mathrm{M}^{+}\right)$; HRMS Calcd. for $\mathrm{C}_{24} \mathrm{H}_{21} \mathrm{~F}_{3} \mathrm{O}_{3}\left(\mathrm{M}^{+}\right)$: 414.1443. Found: 414.1437.

(S)-(2-bromophenyl)phenylmethyl (S)-3,3,3-trifluoro-2-methoxy-2-phenylpropanoate ((S)-MTPA ester of 8)

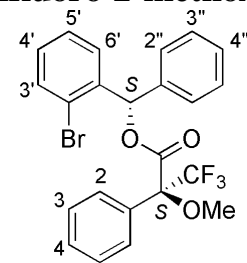

colorless oil; $[\alpha]_{\mathrm{D}}{ }^{28}-17.3\left(c=0.7\right.$ in $\left.\mathrm{CHCl}_{3}\right)$; IR (neat): $v=1752(\mathrm{C}=\mathrm{O}) \mathrm{cm}^{-1} ;{ }^{1} \mathrm{H}$ NMR $\left(500 \mathrm{MHz}, \mathrm{CDCl}_{3}\right): \delta=$ $3.50\left(\mathrm{~d}, J=1.1 \mathrm{~Hz}, 3 \mathrm{H} ; \mathrm{OCH}_{3}\right), 7.18\left(\mathrm{ddd}, J=1.5,7.7,7.8 \mathrm{~Hz}, 1 \mathrm{H} ; \mathrm{H}^{5}\right), 7.26-7.30\left(\mathrm{~m}, 5 \mathrm{H} ; \mathrm{H}^{2}, \mathrm{H}^{3}, \mathrm{H}^{4}\right)$, $7.29-7.34\left(\mathrm{~m}, 2 \mathrm{H} ; \mathrm{H}^{3 \prime}\right), 7.31$ (ddd, $\left.J=1.5,7.7,7.8 \mathrm{~Hz}, 1 \mathrm{H} ; \mathrm{H}^{4}\right), 7.38\left(\mathrm{ddd}, J=2.1,7.9,8.1 \mathrm{~Hz}, 1 \mathrm{H} ; \mathrm{H}^{4}\right.$ ),

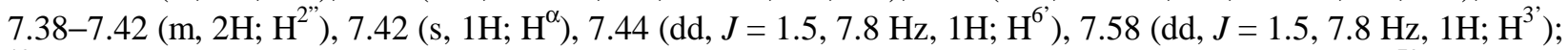
${ }^{19}$ F NMR (254 MHz, CDCl $)$ ): $\delta=-71.88$ (s, 3F); MS: $m / z: 478,480\left(\mathrm{M}^{+}\right)$; HRMS Calcd. for $\mathrm{C}_{23} \mathrm{H}_{18}{ }^{79} \mathrm{BrF}_{3} \mathrm{O}_{3}$ $\left(\mathrm{M}^{+}\right)$: 478.0391. Found: 478.0418. Calcd. for $\mathrm{C}_{23} \mathrm{H}_{18}{ }^{81} \mathrm{BrF}_{3} \mathrm{O}_{3}\left(\mathrm{M}^{+}\right)$: 480.0371. Found: 480.0389.

$(S)$-(2-bromophenyl)phenylmethyl $(R)$-3,3,3-trifluoro-2-methoxy-2-phenylpropanoate $((R)$-MTPA ester of 8)

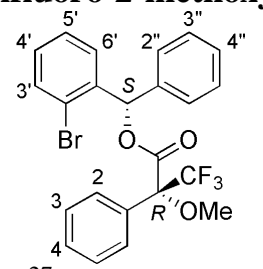

colorless solid (from $\mathrm{CHCl}_{3}$ ); M.p. $63-65{ }^{\circ} \mathrm{C} ;[\alpha]_{\mathrm{D}}{ }^{27}+35.9\left(c=1.1\right.$ in $\left.\mathrm{CHCl}_{3}\right)$; IR (neat): $v=1751(\mathrm{C}=\mathrm{O}) \mathrm{cm}^{-1} ;{ }^{1} \mathrm{H}$ $\operatorname{NMR}\left(500 \mathrm{MHz}, \mathrm{CDCl}_{3}\right): \delta=3.45\left(\mathrm{~s}, 3 \mathrm{H} ; \mathrm{OCH}_{3}\right), 7.16\left(\mathrm{dd}, J=7.5,8.1 \mathrm{~Hz}, 1 \mathrm{H} ; \mathrm{H}^{5}\right), 7.23(\mathrm{dd}, J=7.5,7.7 \mathrm{~Hz}$, $\left.1 \mathrm{H} ; \mathrm{H}^{4}\right), 7.27\left(\mathrm{~d}, J=8.1 \mathrm{~Hz}, 1 \mathrm{H} ; \mathrm{H}^{6}\right), 7.30\left(\mathrm{dd}, J=7.6,8.3 \mathrm{~Hz}, 2 \mathrm{H} ; \mathrm{H}^{3 \prime}\right), 7.30-7.40(\mathrm{~m}, 7 \mathrm{H}), 7.37(\mathrm{~d}, J=7.6 \mathrm{~Hz}$, $\left.2 \mathrm{H} ; \mathrm{H}^{2 "}\right), 7.56\left(\mathrm{~d}, J=7.7 \mathrm{~Hz}, 1 \mathrm{H} ; \mathrm{H}^{3}\right) ;{ }^{3}{ }^{\mathrm{F}} \mathrm{NMR}\left(254 \mathrm{MHz}, \mathrm{CDCl}_{3}\right): \delta=-71.86(\mathrm{~s}, 3 \mathrm{~F}) ; \mathrm{MS}: m / z: 478,480\left(\mathrm{M}^{+}\right)$; HRMS Calcd. for $\mathrm{C}_{23} \mathrm{H}_{18}{ }^{79} \mathrm{BrF}_{3} \mathrm{O}_{3}\left(\mathrm{M}^{+}\right)$: 478.0392. Found: 478.0404. Calcd. for $\mathrm{C}_{23} \mathrm{H}_{18}{ }^{81} \mathrm{BrF}_{3} \mathrm{O}_{3}\left(\mathrm{M}^{+}\right): 480.0371$. Found: 480.0350 .

(S)-(2-chlorophenyl)phenylmethyl $(\boldsymbol{R})$-3,3,3-trifluoro-2-methoxy-2-phenylpropanoate $((R)$-MTPA ester of 9) 


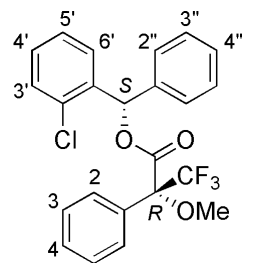

colorless oil; $[\alpha]_{\mathrm{D}}^{30}+45.5\left(c=0.5\right.$ in $\left.\mathrm{CHCl}_{3}\right)$; IR (neat): $v=1753(\mathrm{C}=\mathrm{O}) \mathrm{cm}^{-1} ;{ }^{1} \mathrm{H}$ NMR $\left(500 \mathrm{MHz}, \mathrm{CDCl}_{3}\right): \delta=$ $3.49\left(\mathrm{~d}, J=1.1 \mathrm{~Hz}, 3 \mathrm{H} ; \mathrm{OCH}_{3}\right), 7.19\left(\mathrm{ddd}, J=1.3,7.6,7.7 \mathrm{~Hz}, 1 \mathrm{H} ; \mathrm{H}^{5^{\prime}}\right), 7.24\left(\mathrm{ddd}, J=1.7,7.5,7.7 \mathrm{~Hz}, 1 \mathrm{H} ; \mathrm{H}^{4}\right)$, $7.28\left(\mathrm{dd}, J=1.7,7.6 \mathrm{~Hz}, 1 \mathrm{H} ; \mathrm{H}^{6}\right), 7.36\left(\mathrm{dd}, J=1.3,7.5 \mathrm{~Hz}, 1 \mathrm{H} ; \mathrm{H}^{3}\right), 7.28-7.40(\mathrm{~m}, 10 \mathrm{H}), 7.43\left(\mathrm{~s}, 1 \mathrm{H}, \mathrm{H}^{\alpha}\right) ;{ }^{\prime} \mathrm{F}^{\prime}$ NMR (254 MHz, $\left.\mathrm{CDCl}_{3}\right): \delta=-71.86(\mathrm{~s}, 3 \mathrm{~F})$; MS: $m / z: 434,436\left(\mathrm{M}^{+}\right)$; HRMS Calcd. for $\mathrm{C}_{23} \mathrm{H}_{18}{ }^{35} \mathrm{ClF}_{3} \mathrm{O}_{3}\left(\mathrm{M}^{+}\right)$: 434.0897. Found: 434.0883. Calcd. for $\mathrm{C}_{23} \mathrm{H}_{18}{ }^{37} \mathrm{ClF}_{3} \mathrm{O}_{3}\left(\mathrm{M}^{+}\right)$: 436.0867 . Found: 436.0916 .

(S)-(2-chlorophenyl)phenylmethyl (S)-3,3,3-trifluoro-2-methoxy-2-phenylpropanoate $((S)$-MTPA ester of 9)

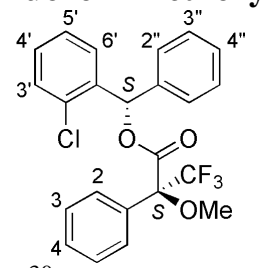

colorless solid (from $\mathrm{CHCl}_{3}$ ); M.p. $78-80{ }^{\circ} \mathrm{C} ;[\alpha]_{\mathrm{D}}{ }^{30}-15.1\left(c=1.0\right.$ in $\left.\mathrm{CHCl}_{3}\right)$; IR $(\mathrm{KBr}): v=1745(\mathrm{C}=\mathrm{O}) \mathrm{cm}^{-1}$; ${ }^{1} \mathrm{H}$ NMR $\left(500 \mathrm{MHz}, \mathrm{CDCl}_{3}\right): \delta=3.50\left(\mathrm{~s}, 3 \mathrm{H} ; \mathrm{OCH}_{3}\right), 7.25-7.27\left(\mathrm{~m}, 1 \mathrm{H} ; \mathrm{H}^{5}\right), 7.26-7.30\left(\mathrm{~m}, 1 \mathrm{H} ; \mathrm{H}^{4}\right), 7.37-7.41$ $\left(\mathrm{m}, 1 \mathrm{H} ; \mathrm{H}^{6}\right), 7.42-7.45\left(\mathrm{~m}, 1 \mathrm{H} ; \mathrm{H}^{3^{\prime}}\right) 7.25-7.45(\mathrm{~m}, 11 \mathrm{H}) ;{ }^{19} \mathrm{~F} \mathrm{NMR}\left(254 \mathrm{MHz}, \mathrm{CDCl}_{3}\right): \delta=-71.90(\mathrm{~s}, 3 \mathrm{~F})$; MS: $m / z: 434,436\left(\mathrm{M}^{+}\right)$; HRMS Calcd. for $\mathrm{C}_{23} \mathrm{H}_{18}{ }^{35} \mathrm{ClF}_{3} \mathrm{O}_{3}\left(\mathrm{M}^{+}\right)$: 434.0896. Found: 434.0872. Calcd. for $\mathrm{C}_{23} \mathrm{H}_{18}{ }^{37} \mathrm{ClF}_{3} \mathrm{O}_{3}\left(\mathrm{M}^{+}\right)$: 436.0867. Found: 436.0903 .

(S)-(2-fluorophenyl)phenylmethyl (S)-3,3,3-trifluoro-2-methoxy-2-phenylpropanoate ((S)-MTPA ester of 10)

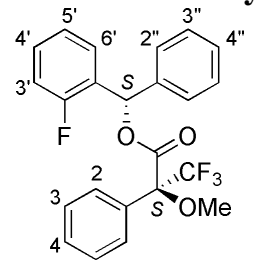

colorless solid (from $\mathrm{CHCl}_{3}$ ); M.p. $48-50{ }^{\circ} \mathrm{C} ;[\alpha]_{\mathrm{D}}{ }^{29}-21.9\left(c=0.7\right.$ in $\left.\mathrm{CHCl}_{3}\right)$; IR $(\mathrm{KBr}): v=1754(\mathrm{C}=\mathrm{O}) \mathrm{cm}^{-1}$; ${ }^{1} \mathrm{H}$ NMR $\left(500 \mathrm{MHz}, \mathrm{CDCl}_{3}\right): \delta=3.52\left(\mathrm{~s}, 3 \mathrm{H} ; \mathrm{OCH}_{3}\right), 7.05-7.13(\mathrm{~m}, 2 \mathrm{H}), 7.24-7.35(\mathrm{~m}, 10 \mathrm{H}), 7.37-7.42(\mathrm{~m}$, $3 \mathrm{H}) ;{ }^{19} \mathrm{~F}$ NMR $\left(254 \mathrm{MHz}, \mathrm{CDCl}_{3}\right): \delta=-71.92(\mathrm{~s}, 3 \mathrm{~F}),-117.13(\mathrm{ddd}, J=5.1,5.1,10.2 \mathrm{~Hz}, 1 \mathrm{~F}) ; \mathrm{MS}: m / z: 418$ $\left(\mathrm{M}^{+}\right)$; HRMS Calcd. for $\mathrm{C}_{23} \mathrm{H}_{18} \mathrm{~F}_{4} \mathrm{O}_{3}\left(\mathrm{M}^{+}\right)$: 418.1192. Found: 418.1156 .

(S)-(2-fluorophenyl)phenylmethyl $(\boldsymbol{R})$-3,3,3-trifluoro-2-methoxy-2-phenylpropanoate $((R)$-MTPA ester of 10)

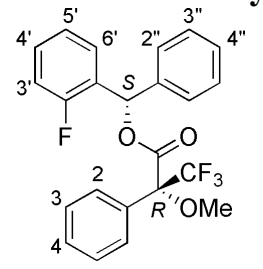

colorless oil; $[\alpha]_{\mathrm{D}}{ }^{28}+38.6\left(c=0.4\right.$ in $\left.\mathrm{CHCl}_{3}\right)$; IR (neat): $v=1753(\mathrm{C}=\mathrm{O}) \mathrm{cm}^{-1} ;{ }^{1} \mathrm{H}$ NMR $\left(500 \mathrm{MHz}, \mathrm{CDCl}_{3}\right): \delta=$ $3.50\left(\mathrm{~d}, J=1.1 \mathrm{~Hz}, 3 \mathrm{H} ; \mathrm{OCH}_{3}\right), 7.01-7.08(\mathrm{~m}, 2 \mathrm{H}), 7.21(\mathrm{dd}, J=7.6,7.6 \mathrm{~Hz}, 1 \mathrm{H}), 7.24-7.40(\mathrm{~m}, 12 \mathrm{H}) ;{ }^{19} \mathrm{~F} \mathrm{NMR}$ $\left(254 \mathrm{MHz}, \mathrm{CDCl}_{3}\right): \delta=-71.93(\mathrm{~s}, 3 \mathrm{~F}),-117.33(\mathrm{~m}, 1 \mathrm{~F}) ; \mathrm{MS} \mathrm{m} / \mathrm{z}: 418\left(\mathrm{M}^{+}\right) ;$HRMS Calcd. for $\mathrm{C}_{23} \mathrm{H}_{18} \mathrm{~F}_{4} \mathrm{O}_{3}\left(\mathrm{M}^{+}\right)$: 418.1193. Found: 418.1212.

Physical and Spectral Data for the (S)- and (R)-CFTA Esters of $\alpha$-Monodeuterated Benzyl Alcohols 13-17.

$(S)-\left[1-^{2} \mathbf{H}\right]$-phenylmethyl (S)-2-cyano-2-fluoro-2-(4-methylphenyl)acetate ((S)-CFTA ester of 13) 


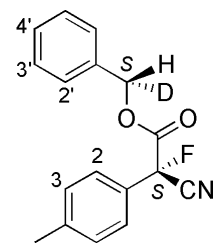

colorless solid; M.p. $36-39^{\circ} \mathrm{C}$; $[\alpha]_{\mathrm{D}}{ }^{26}+0.4\left(c=1.6\right.$ in $\left.\mathrm{CHCl}_{3}\right)$; IR $(\mathrm{KBr}): v=1770(\mathrm{C}=\mathrm{O}), 2251(\mathrm{CN}) \mathrm{cm}^{-1} ;{ }^{1} \mathrm{H}$ $\operatorname{NMR}\left(500 \mathrm{MHz}, \mathrm{CDCl}_{3}\right): \delta=2.39\left(\mathrm{~s}, 3 \mathrm{H} ; \mathrm{CH}_{3}\right), 5.28\left(\mathrm{~s}, 1 \mathrm{H} ; \mathrm{H}^{\alpha}\right), 7.24\left(\mathrm{~d}, J=8.0 \mathrm{~Hz}, 2 \mathrm{H} ; \mathrm{H}^{3}\right), 7.24-7.28(\mathrm{~m}$, $2 \mathrm{H}), 7.32-7.36(\mathrm{~m}, 3 \mathrm{H}), 7.47\left(\mathrm{~d}, J=8.0 \mathrm{~Hz}, 2 \mathrm{H} ; \mathrm{H}^{2}\right) ;{ }^{2} \mathrm{D} \mathrm{NMR}\left(61 \mathrm{MHz}, \mathrm{CHCl}_{3}\right): \delta=5.31$ (brd, $\left.J=1.7 \mathrm{~Hz}, 1 \mathrm{D}\right)$; ${ }^{19}$ F NMR (254 MHz, CDCl 3 ): $\delta=-144.40(\mathrm{~s}, 1 \mathrm{~F})$; MS: $m / z: 284\left(\mathrm{M}^{+}\right)$; HRMS Calcd. For $\mathrm{C}_{17} \mathrm{H}_{13} \mathrm{DFNO}_{2}\left(\mathrm{M}^{+}\right)$: 284.1070. Found: 284.1076.

$(S)-\left[1-^{2} \mathbf{H}\right]$-phenylmethyl $(R)$-2-cyano-2-fluoro-2-(4-methylphenyl)acetate $((R)$-CFTA ester of 13)

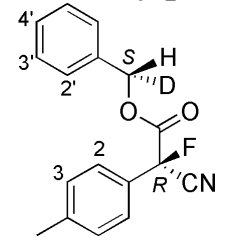

colorless solid (from $\left.\mathrm{CHCl}_{3}\right)$; M.p. $22^{\circ} \mathrm{C}$; $[\alpha]_{\mathrm{D}}{ }^{26}+0.9\left(c=1.6\right.$ in $\left.\mathrm{CHCl}_{3}\right)$; IR $(\mathrm{KBr}): v=1773(\mathrm{C}=\mathrm{O}), 2254(\mathrm{CN})$ $\mathrm{cm}^{-1} ;{ }^{1} \mathrm{H}$ NMR $\left(500 \mathrm{MHz}, \mathrm{CDCl}_{3}\right): \delta=2.39\left(\mathrm{~s}, 3 \mathrm{H} ; \mathrm{CH}_{3}\right), 5.25\left(\mathrm{~s}, 1 \mathrm{H} ; \mathrm{H}^{\alpha}\right), 7.24-7.28(\mathrm{~m}, 2 \mathrm{H}), 7.25(\mathrm{~d}, J=8.0$ $\left.\mathrm{Hz}, 2 \mathrm{H} ; \mathrm{H}^{3}\right), 7.32-7.35(\mathrm{~m}, 3 \mathrm{H}), 7.48\left(\mathrm{~d}, J=8.0 \mathrm{~Hz}, 2 \mathrm{H} ; \mathrm{H}^{2}\right) ;{ }^{2} \mathrm{D}$ NMR $\left(61 \mathrm{MHz}, \mathrm{CHCl}_{3}\right): \delta=5.28(\mathrm{brd}, J=1.7$ $\mathrm{Hz}, 1 \mathrm{D}) ;{ }^{19} \mathrm{~F}$ NMR $\left(254 \mathrm{MHz}, \mathrm{CDCl}_{3}\right): \delta=-144.43$ (s, 1F); MS: $m / z: 284\left(\mathrm{M}^{+}\right)$; HRMS Calcd. For $\mathrm{C}_{17} \mathrm{H}_{13} \mathrm{DFNO}_{2}$ $\left(\mathrm{M}^{+}\right): 284.1070$. Found: 284.1084.

$(S)$-[1- ${ }^{2}$ H]-(4-methylphenyl)methyl (S)-2-cyano-2-fluoro-2-(4-methylphenyl)acetate $((S)$-CFTA ester of 14)

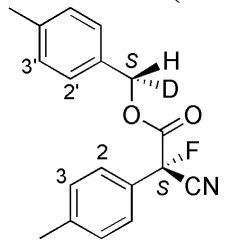

colorless solid (from $\left.\mathrm{CHCl}_{3}\right)$; M.p. $22{ }^{\circ} \mathrm{C} ;[\alpha]_{\mathrm{D}}{ }^{26}+2.9\left(c=1.1\right.$ in $\left.\mathrm{CHCl}_{3}\right)$; IR $(\mathrm{KBr}): v=1767(\mathrm{C}=\mathrm{O}), 2254(\mathrm{CN})$ $\mathrm{cm}^{-1} ;{ }^{1} \mathrm{H}$ NMR $\left(500 \mathrm{MHz}, \mathrm{CDCl}_{3}\right): \delta=2.35\left(\mathrm{~s}, 3 \mathrm{H} ;>\mathrm{CH}-\mathrm{Ar}-\mathrm{CH}_{3}\right), 2.39\left(\mathrm{~s}, 3 \mathrm{H} ;>\mathrm{CF}-\mathrm{Ar}_{-}-\mathrm{CH}_{3}\right), 5.25\left(\mathrm{~s}, 1 \mathrm{H} ; \mathrm{H}^{\alpha}\right)$, $7.13-7.18(\mathrm{~m}, 4 \mathrm{H}), 7.24\left(\mathrm{~d}, J=8.0 \mathrm{~Hz}, 2 \mathrm{H} ; \mathrm{H}^{3}\right), 7.47\left(\mathrm{~d}, J=8.0 \mathrm{~Hz}, 2 \mathrm{H} ; \mathrm{H}^{2}\right) ;{ }^{2} \mathrm{D}$ NMR $\left(61 \mathrm{MHz}, \mathrm{CHCl}_{3}\right): \delta=$ 5.23 (brd, $J=1.5 \mathrm{~Hz}, 1 \mathrm{D}) ;{ }^{19} \mathrm{~F}$ NMR $\left(254 \mathrm{MHz}, \mathrm{CDCl}_{3}\right): \delta=-144.43$ (s, 1F); MS: $m / z: 298\left(\mathrm{M}^{+}\right)$; HRMS Calcd. For $\mathrm{C}_{18} \mathrm{H}_{15} \mathrm{DFNO}_{2}\left(\mathrm{M}^{+}\right)$: 298.1227. Found: 298.1220.

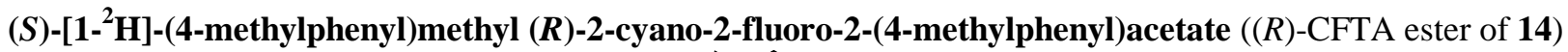

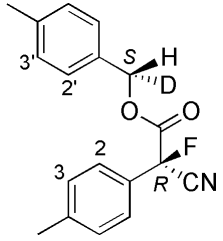

colorless oil; $[\alpha]_{\mathrm{D}}{ }^{26}-2.2\left(c=0.7\right.$ in $\left.\mathrm{CHCl}_{3}\right)$; IR (neat): $v=1772(\mathrm{C}=\mathrm{O}), 2253(\mathrm{CN}) \mathrm{cm}^{-1} ;{ }^{1} \mathrm{H} \mathrm{NMR}(500 \mathrm{MHz}$, $\left.\mathrm{CDCl}_{3}\right): \delta=2.35\left(\mathrm{~s}, 3 \mathrm{H} ;>\mathrm{CH}-\mathrm{Ar}-\mathrm{CH}_{3}\right), 2.39\left(\mathrm{~s}, 3 \mathrm{H} ;>\mathrm{CF}-\mathrm{Ar}-\mathrm{CH}_{3}\right), 5.20\left(\mathrm{~s}, 1 \mathrm{H} ; \mathrm{H}^{\alpha}\right), 7.13-7.18(\mathrm{~m}, 4 \mathrm{H}), 7.24(\mathrm{~d}$, $\left.J=8.0 \mathrm{~Hz}, 2 \mathrm{H} ; \mathrm{H}^{3}\right), 7.47\left(\mathrm{~d}, J=8.0 \mathrm{~Hz}, 2 \mathrm{H} ; \mathrm{H}^{2}\right) ;{ }^{2} \mathrm{D} \mathrm{NMR}\left(61 \mathrm{MHz}, \mathrm{CHCl}_{3}\right): \delta=5.28(\mathrm{brd}, J=1.5 \mathrm{~Hz}, 1 \mathrm{D}) ;{ }^{19} \mathrm{~F}$ $\operatorname{NMR}\left(254 \mathrm{MHz}, \mathrm{CDCl}_{3}\right): \delta=-144.43$ (s, 1F); MS: $m / z: 298\left(\mathrm{M}^{+}\right)$; HRMS Calcd. For $\mathrm{C}_{18} \mathrm{H}_{15} \mathrm{DFNO}_{2}\left(\mathrm{M}^{+}\right)$: 298.1227. Found: 298.1223.

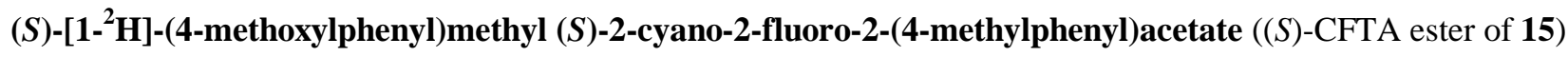




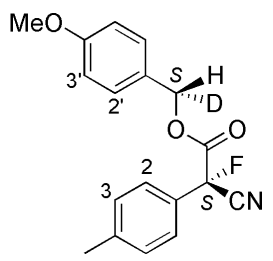

colorless solid; M.p. $37-40{ }^{\circ} \mathrm{C} ;[\alpha]_{\mathrm{D}}{ }^{28}+3.8\left(c=1.0\right.$ in $\left.\mathrm{CHCl}_{3}\right)$; IR $(\mathrm{KBr}): \mathrm{v}=1764(\mathrm{C}=\mathrm{O}), 2258(\mathrm{CN}) \mathrm{cm}^{-1} ;{ }^{1} \mathrm{H}$ $\operatorname{NMR}\left(500 \mathrm{MHz}, \mathrm{CDCl}_{3}\right): \delta=2.39\left(\mathrm{~s}, 3 \mathrm{H} ; \mathrm{CH}_{3}\right), 3.81\left(\mathrm{~s}, 3 \mathrm{H} ; \mathrm{OCH}_{3}\right), 5.22\left(\mathrm{~s}, 1 \mathrm{H} ; \mathrm{H}^{\alpha}\right), 6.84-6.87(\mathrm{~m}, 2 \mathrm{H})$, 7.21-7.24 (m, 2H), $7.24\left(\mathrm{~d}, J=8.0 \mathrm{~Hz}, 2 \mathrm{H} ; \mathrm{H}^{3}\right), 7.45\left(\mathrm{~d}, J=8.0 \mathrm{~Hz}, 2 \mathrm{H} ; \mathrm{H}^{2}\right) ;{ }^{2} \mathrm{D}$ NMR $\left(61 \mathrm{MHz}, \mathrm{CHCl}_{3}\right): \delta=$ 5.22 (brs, 1D); ${ }^{19} \mathrm{~F} \mathrm{NMR}\left(254 \mathrm{MHz}, \mathrm{CDCl}_{3}\right): \delta=-144.32$ (s, $\left.1 \mathrm{~F}\right) ; \mathrm{MS}: m / z: 314\left(\mathrm{M}^{+}\right) ; \mathrm{HRMS}$ Calcd. For $\mathrm{C}_{18} \mathrm{H}_{15} \mathrm{DFNO}_{3}\left(\mathrm{M}^{+}\right): 314.1176$. Found: 314.1179 .

$(S)$-[1-' $\mathbf{H}]$-(4-methoxylphenyl)methyl (R)-2-cyano-2-fluoro-2-(4-methylphenyl)acetate ((R)-CFTA ester of 15)

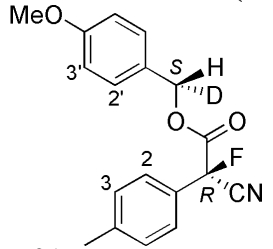

colorless solid (from $\mathrm{CHCl}_{3}$ ); M.p. $37-40{ }^{\circ} \mathrm{C}$; $[\alpha]_{\mathrm{D}}{ }^{25}-1.5\left(c=1.1\right.$ in $\left.\mathrm{CHCl}_{3}\right)$; IR $(\mathrm{KBr}): v=1764(\mathrm{C}=\mathrm{O}), 2258$ $(\mathrm{CN}) \mathrm{cm}^{-1} ;{ }^{1} \mathrm{H}$ NMR $\left(500 \mathrm{MHz}, \mathrm{CDCl}_{3}\right): \delta=2.39\left(\mathrm{~s}, 3 \mathrm{H} ; \mathrm{CH}_{3}\right), 3.81\left(\mathrm{~s}, 3 \mathrm{H} ; \mathrm{OCH}_{3}\right), 5.19\left(\mathrm{~s}, 1 \mathrm{H} ; \mathrm{H}^{\alpha}\right), 6.84-6.87$ $(\mathrm{m}, 2 \mathrm{H}), 7.21-7.24(\mathrm{~m}, 2 \mathrm{H}), 7.24\left(\mathrm{~d}, J=8.0 \mathrm{~Hz}, 2 \mathrm{H} ; \mathrm{H}^{3}\right), 7.45\left(\mathrm{~d}, J=8.0 \mathrm{~Hz}, 2 \mathrm{H} ; \mathrm{H}^{2}\right) ;{ }^{2} \mathrm{D}$ NMR $(61 \mathrm{MHz}$, $\mathrm{CHCl}_{3}$ ): $\delta=5.24$ (brs, 1D); $\left.{ }^{19} \mathrm{~F} \mathrm{NMR} \mathrm{(254} \mathrm{MHz,} \mathrm{CDCl}_{3}\right): \delta=-144.30$ (s, 1F); MS: $m / z: 314\left(\mathrm{M}^{+}\right)$; HRMS Calcd. For $\mathrm{C}_{18} \mathrm{H}_{15} \mathrm{DFNO}_{3}\left(\mathrm{M}^{+}\right)$: 314.1176. Found: 314.1190 .

$(S)-\left[1-{ }^{2} \mathrm{H}\right]-[4-($ trifluoromethyl)phenyl]methyl (S)-2-cyano-2-fluoro-2-(4-methylphenyl)-acetate $((S)$-CFTA ester of 16)

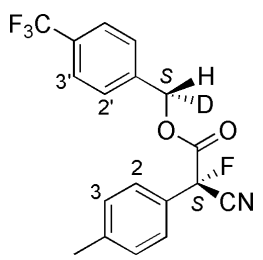

colorless oil; $[\alpha]_{\mathrm{D}}{ }^{26}-0.8\left(c=0.1\right.$ in $\left.\mathrm{CHCl}_{3}\right)$; IR (neat): $v=1777(\mathrm{C}=\mathrm{O}), 2256(\mathrm{CN}) \mathrm{cm}^{-1} ;{ }^{1} \mathrm{H} \mathrm{NMR}(500 \mathrm{MHz}$, $\left.\mathrm{CDCl}_{3}\right): \delta=2.40\left(\mathrm{~s}, 3 \mathrm{H} ; \mathrm{CH}_{3}\right), 5.32\left(\mathrm{~s}, 1 \mathrm{H} ; \mathrm{H}^{\alpha}\right), 7.24-7.28(\mathrm{~m}, 2 \mathrm{H}), 7.36\left(\mathrm{~d}, J=8.0 \mathrm{~Hz}, 2 \mathrm{H} ; \mathrm{H}^{3}\right), 7.48(\mathrm{~d}, J=7.5$ $\mathrm{Hz}, 2 \mathrm{H}), 7.59\left(\mathrm{~d}, J=8.0 \mathrm{~Hz}, 2 \mathrm{H} ; \mathrm{H}^{2}\right) ;{ }^{2} \mathrm{D}$ NMR $\left(61 \mathrm{MHz}, \mathrm{CHCl}_{3}\right): \delta=5.33$ (brs, $\left.1 \mathrm{D}\right) ;{ }^{19} \mathrm{~F} \mathrm{NMR}(254 \mathrm{MHz}$, $\left.\mathrm{CDCl}_{3}\right): \delta=-63.34$ (s, 3F), -144.86 (s, 1F); MS: $m / z: 352\left(\mathrm{M}^{+}\right)$; HRMS Calcd. for $\mathrm{C}_{18} \mathrm{H}_{12} \mathrm{DF}_{4} \mathrm{NO}_{2}\left(\mathrm{M}^{+}\right)$: 352.0944. Found: 352.0956.

$(S)$-[1- $\left.{ }^{2} \mathrm{H}\right]$-[4-(trifluoromethyl)phenyl]methyl (R)-2-cyano-2-fluoro-2-(4-methylphenyl)-acetate $((R)$-CFTA ester of 16)

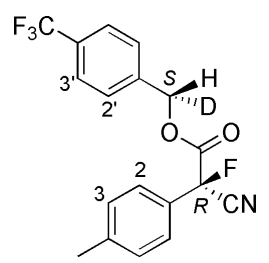

colorless oil; $[\alpha]_{\mathrm{D}}{ }^{26}+2.4\left(c=0.2\right.$ in $\left.\mathrm{CHCl}_{3}\right)$; IR (neat): $v=1777(\mathrm{C}=\mathrm{O}), 2253(\mathrm{CN}) \mathrm{cm}^{-1} ;{ }^{1} \mathrm{H}$ NMR $(500 \mathrm{MHz}$, $\left.\mathrm{CDCl}_{3}\right): \delta=2.40\left(\mathrm{~s}, 3 \mathrm{H} ; \mathrm{CH}_{3}\right), 5.30\left(\mathrm{~s}, 1 \mathrm{H} ; \mathrm{H}^{\alpha}\right), 7.24-7.28(\mathrm{~m}, 2 \mathrm{H}), 7.36\left(\mathrm{~d}, J=8.0 \mathrm{~Hz}, 2 \mathrm{H} ; \mathrm{H}^{3}\right), 7.48(\mathrm{~d}, J=7.5$ $\mathrm{Hz}, 2 \mathrm{H}), 7.59\left(\mathrm{~d}, J=8.0 \mathrm{~Hz}, 2 \mathrm{H} ; \mathrm{H}^{2}\right) ;{ }^{2} \mathrm{D}$ NMR $\left(61 \mathrm{MHz}, \mathrm{CHCl}_{3}\right): \delta=5.35$ (brs, $\left.1 \mathrm{D}\right) ;{ }^{19} \mathrm{~F} \mathrm{NMR}(254 \mathrm{MHz}$, $\left.\mathrm{CDCl}_{3}\right): \delta=-144.40(\mathrm{~s}, 1 \mathrm{~F})$; MS: $m / z: 352\left(\mathrm{M}^{+}\right)$; HRMS Calcd. for $\mathrm{C}_{18} \mathrm{H}_{12} \mathrm{DF}_{4} \mathrm{NO}_{2}\left(\mathrm{M}^{+}\right): 352.0944$. Found: 352.0930 .

$(S)$-[1- ${ }^{2}$ H]-(4-bromophenyl)methyl $(S)$-2-cyano-2-fluoro-2-(4-methylphenyl)acetate ((S)-CFTA ester of 17) 
colorless solid (from $\mathrm{CHCl}_{3}$ ); M.p. $30-32{ }^{\circ} \mathrm{C}$; $[\alpha]_{\mathrm{D}}{ }^{26}+0.5\left(\right.$ c 1.7 in $\left.\mathrm{CHCl}_{3}\right)$; IR $(\mathrm{KBr}): v=1769(\mathrm{C}=\mathrm{O}), 2253(\mathrm{CN})$ $\mathrm{cm}^{-1} ;{ }^{1} \mathrm{H}$ NMR $\left(500 \mathrm{MHz}, \mathrm{CDCl}_{3}\right): \delta=2.40\left(\mathrm{~s}, 3 \mathrm{H} ; \mathrm{CH}_{3}\right), 5.22\left(\mathrm{~s}, 1 \mathrm{H} ; \mathrm{H}^{\alpha}\right), 7.11-7.14(\mathrm{~m}, 2 \mathrm{H}), 7.25(\mathrm{~d}, J=8.0 \mathrm{~Hz}$, $\left.2 \mathrm{H} ; \mathrm{H}^{3}\right), 7.46\left(\mathrm{~d}, J=8.0 \mathrm{~Hz}, 2 \mathrm{H} ; \mathrm{H}^{2}\right), 7.45-7.48(\mathrm{~m}, 2 \mathrm{H}) ;{ }^{2} \mathrm{D} \mathrm{NMR}\left(61 \mathrm{MHz}, \mathrm{CHCl}_{3}\right): \delta=5.23(\mathrm{brs}, 1 \mathrm{D}) ;{ }^{19} \mathrm{~F}$ NMR (254 MHz, CDCl $): \delta=-144.71(\mathrm{~s}, 1 \mathrm{~F})$; MS: $m / z: 362$, $364\left(\mathrm{M}^{+}\right)$; HRMS Calcd. For $\mathrm{C}_{17} \mathrm{H}_{12} \mathrm{D}^{79} \mathrm{BrFNO}_{2}$ $\left(\mathrm{M}^{+}\right)$: 362.0175. Found: 362.0191. Calcd. for $\mathrm{C}_{17} \mathrm{H}_{12} \mathrm{D}^{81} \mathrm{BrFNO}_{2}\left(\mathrm{M}^{+}\right)$: 364.0156. Found: 364.0139.

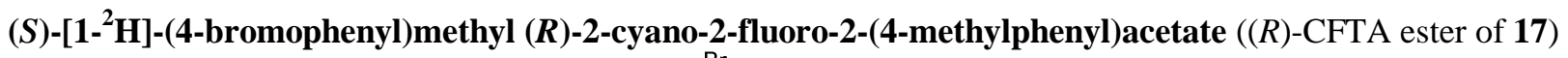

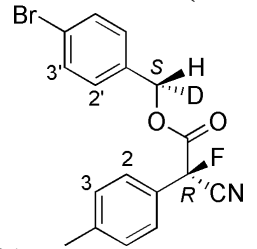

yellow solid (from $\left.\mathrm{CHCl}_{3}\right)$; M.p. $30-32{ }^{\circ} \mathrm{C}$; $[\alpha]_{\mathrm{D}}{ }^{25}+3.4\left(c=0.3\right.$ in $\left.\mathrm{CHCl}_{3}\right)$; IR $(\mathrm{KBr}): \mathrm{v}=1774(\mathrm{C}=\mathrm{O}), 2256(\mathrm{CN})$ $\mathrm{cm}^{-1} ;{ }^{1} \mathrm{H}$ NMR $\left(500 \mathrm{MHz}, \mathrm{CDCl}_{3}\right): \delta=2.40\left(\mathrm{~s}, 3 \mathrm{H} ; \mathrm{CH}_{3}\right), 5.20\left(\mathrm{~s}, 1 \mathrm{H} ; \mathrm{H}^{\alpha}\right), 7.11-7.14(\mathrm{~m}, 2 \mathrm{H}), 7.25(\mathrm{~d}, J=8.0 \mathrm{~Hz}$, $\left.2 \mathrm{H} ; \mathrm{H}^{3}\right), 7.46\left(\mathrm{~d}, J=8.0 \mathrm{~Hz}, 2 \mathrm{H} ; \mathrm{H}^{2}\right), 7.45-7.48(\mathrm{~m}, 2 \mathrm{H}) ;{ }^{2} \mathrm{D}$ NMR $\left(61 \mathrm{MHz}, \mathrm{CHCl}_{3}\right): \delta=5.24$ (brs, $\left.1 \mathrm{D}\right) ;{ }^{19} \mathrm{~F}$ NMR (254 MHz, $\left.\mathrm{CDCl}_{3}\right): \delta=-144.71(\mathrm{~s}, 1 \mathrm{~F}) ; \mathrm{MS}: \mathrm{m} / z: 362,364\left(\mathrm{M}^{+}\right)$; HRMS Calcd. For $\mathrm{C}_{17} \mathrm{H}_{12} \mathrm{D}^{79} \mathrm{BrFNO}_{2}$ $\left(\mathrm{M}^{+}\right)$: 362.0175. Found: 362.0176. Calcd. for $\mathrm{C}_{17} \mathrm{H}_{12} \mathrm{D}^{81} \mathrm{BrFNO}_{2}\left(\mathrm{M}^{+}\right)$: 364.0156. Found: 364.0169. 